SERVIÇO DE PÓS-GRADUAÇÃO DO ICMC-USP

Data de Depósito: 06/02/2004

Assinatura: Ana Paula lamiraio irelare

\title{
Difeomorfismos do produto de esferas e mergulho de esferas neste produto
}

\author{
Mario Henrique Andrade Claudio ${ }^{1}$
}

Orientador: Prof. Dr. Oziride Manzoli Neto

Dissertação apresentada ao Instituto de Ciências Matemáticas e de Computação da Universidade de São Paulo, como parte dos requisitos para obtenção do título de Mestre em ciências - Área: Matemática.

USP - São Carlos

Fevereiro/2004

${ }^{1}$ Este trabalho teve suporte financeiro da CAPES 
A Comissão Julgadora:

Prof. Dr. Oziride Manzoli Neto

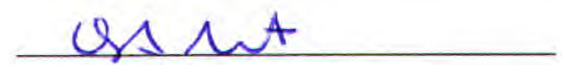

Prof. Dr. José Eduardo Prado Pires de Campos 
Aos meus pais e minhas irmãs. 


\section{Agradecimentos}

Primeiramente agradeço a Deus, que sempre está ao meu lado me guiando pelos caminhos mais seguros, me iluminando e colocando nesse meu caminhar, seus braços sempre a disposição para me carregar e dar descanso quando o fardo da vida faz com que minhas pernas fraquejem.

Ao Prof. Oziride Manzoli Neto, o Dide, pela orientação não apenas neste trabalho mas em todo meu tempo acadêmico, aconselhando-me sempre de maneira a incentivar meu crescimento profissional, e acima de tudo pessoal.

Aos meus pais, Luiz Antonio e Ursulina, e minhas irmãs, Vanessa e Perla, que acreditam e incentivam-me em todos os passos de minha vida, estando sempre ao meu lado. Obrigado por serem meu porto seguro.

A toda minha família, especialmente minhas avós, que colaboraram muito para que esta etapa fosse concluída, com suas orações e ações para meu fortalecimento espiritual e físico durante toda a caminhada.

Aos amigos da minha cidade, Tietê, Sinésio, Eduardo e Luis, aos amigos da graduação e da pós-graduação, Aline, Alex, Fábio, Flávia, Grazielle, Kelly, Luci Any, Mariana, Paula e Romário, que estiveram ao meu lado sempre dispostos a dar o apoio que inúmeras vezes precisei, me levantando, me guiando, me incentivando. Obrigado por acreditarem em mim.

A todas as outras pessoas, professores e funcionários do ICMC, que colaboraram de alguma forma para a realização deste trabalho.

Muito obrigado

a todos! 


\section{Resumo}

Seja $M$ o produto de $n$ cópias da esfera $p$-dimensional $S^{p}$ com $p \geq 1$ e $n \geq 2$. Estudaremos nesta dissertação todos os automorfismos de $H_{p}(M ; \mathbb{Z}) \cong \mathbb{Z}^{n}$ que são realizados por difeomorfismos de $M$, fazendo uma classificação destes. Como uma aplicação, veremos uma classificação completa das esferas $p$-dimensionais mergulhadas suavemente em $M$ a menos de difeomorfismos de $M$. 


\begin{abstract}
Let $M$ be the product of $n$ copies of the $p$-dimensional sphere $S^{p}$ with $p \geq 1$ and $n \geq 2$. We will study in this work all automorphisms of $H_{p}(M ; \mathbb{Z}) \cong \mathbb{Z}^{n}$ which can be realized by a diffeomorphism of $M$, making a classification of them. As an application, we will see a complete classification of smoothly embedded $p$-dimensional spheres in $M$ up to diffeomorphisms of $M$.
\end{abstract}




\section{Sumário}

$\begin{array}{ll}\text { Introdução } & 1\end{array}$

1 Preliminares $\quad 3$

1.1 Älgebra . . . . . . . . . . . . . . . . . . . . . . 3

1.2 Homologia e cohomologia . . . . . . . . . . . . . . . 8

1.3 Homotopia. . . . . . . . . . . . . . . . . . . . 13

1.4 Topologia das variedades diferenciáveis . . . . . . . . . . . . . . 16

1.5 Difeomorfismos do produtos de esferas . . . . . . . . . . . . . . 17

2 Classificação das matrizes que representam automorfismos $\quad 22$

2.1 Lemas básicos . . . . . . . . . . . . . . . . . . . . . 22

2.2 Teorema de classificação das matrizes que representam automorfismos . . . . 28

2.3 Alguns resultados especiais . . . . . . . . . . . . . . . 36

3 Classificação dos mergulhos de esferas 38

3.1 O teorema de classificação de esferas mergulhadas no produto de esferas . . . 38

4 Casos especiais $\quad \mathbf{4 6}$

4.1 Classificação de esferas mergulhadas no produto de esferas, caso $n=2 \ldots 46$

4.2 O caso orientável . . . . . . . . . . . . . . . . 47

$\begin{array}{ll}\text { Referências Bibliográficas } & 50\end{array}$ 


\section{Introdução}

O objetivo desta dissertação é estudar todos os automorfismos de $H_{p}(M ; \mathbb{Z}) \cong \mathbb{Z}^{n}$ que são realizados como homomorfismos induzidos por difeomorfismos de $M$, onde $M=S^{p} \times \ldots \times S^{p}$, o produto de $n$ cópias da $p$-esfera $S^{p}$ com $p \geq 1$ e $n \geq 2$, e classificar mergulhos suaves de $S^{p}$ em $M$. Todo difeomorfismo $f: M \rightarrow M$ induz um automorfismo $f_{*}$ de $H_{p}(M ; \mathbb{Z}) \cong \mathbb{Z}^{n}$, o qual pode ser considerado como um elemento de $G L(n ; \mathbb{Z})=\{A \in M(n \times n ; \mathbb{Z}) ; \operatorname{det} A= \pm 1\}$, o grupo das matrizes de ordem $n$ em $\mathbb{Z}$ com determinante \pm 1 e munido da multiplicação, e seja $D_{p}$ o subgrupo de $G L(n ; \mathbb{Z})$ consistindo de todos os automorfismos que são induzidos por difeomorfismos de $M$.

Em 1962, C.T. Wall [22], em 1967, R.Z. Goldstein [6] e em 1969, H. Sato [18], fizeram um estudo deste problema para o caso $n=2$. O que estudaremos aqui é uma generalização desses resultados feita em Lucas [14], que mostra:

(i) $D_{p}$ coincide com $G L(n ; \mathbb{Z})$ para $p=1,3,7$,

(ii) $D_{p}$ é um subgrupo de índice $2^{\left(n^{2}-n\right) / 2}\left(2^{1}-1\right)\left(2^{2}-1\right) \ldots\left(2^{n}-1\right) / n$ ! para $p$ ímpar com $p \neq 1,3,7$,

(iii) $D_{p}$ é um subgrupo finito de ordem $2^{n} n$ ! para $p$ par.

Em 1968, R.Z. Goldstein [7] fez uma classificação das esferas p-dimensionais mergulhadas suavemente em $M$ a menos de difeomorfismos de $M$, para o caso $n=2$ e $p \geq 4$. A classificação aqui apresentada ê uma generalização deste caso, mais precisamente, se supormos que $p=1$ e $n \geq 4$, ou $p=2,3$ e $n \geq 3$, ou $p \geq 4$ e $n \geq 2$ veremos uma lista de formas padrões de tais mergulhos e que qualquer esfera $p$-dimensional mergulhada suavemente pode ser aplicada continuamente a exatamente uma das formas padrões por um difeomorfismo de $M$.

Apresentamos o trabalho em quatro capitulos como a seguir:

No capítulo 1 enunciamos algumas definições e resultados importantes para o desenvolvimento da dissertação.

No capítulo 2 provaremos o principal resultado deste trabalho, o Teorema 2.2 .1 , que é 
uma generalização do caso $n=2$, feito por Wall e Goldstein, que trata da classificação das matrizes que representam automorfismos de $M$.

No capítulo 3, como uma aplicação do Teorema 2.2 .1 (classificação das matrizes que representam automorfismos), apresentaremos o Teorema 3.1.2, que trata da classificação de esferas mergulhadas.

No capitulo 4, veremos a prova do Teorema 3.1.2 (classificação de esferas mergulhadas feita por Goldstein [7]) quando consideramos $n=2$, e posteriormente analisaremos quais modificações são necessárias ao teorema 3.1.2 quando consideramos as orientações de $S^{p}$ e de $M$. 


\section{Capítulo 1}

\section{Preliminares}

Neste capítulo apresentaremos algumas definições e resultados básicos sobre álgebra, topologia diferencial e algébrica, com aplicações de alguns deles que serão necessários nos capítulos posteriores.

\section{1 Álgebra}

Comecemos relembrado alguns resultados sobre matrizes. Veja Hoffman-Kunze [13] para mais detalhes.

Define-se por operações elementares sobre as linhas de uma matriz $A$ de dimensão $m \times n$ sobre um corpo $F$, as operações:

(i) A transposição de duas quaisquer linhas.

(ii) A multiplicação de uma linha por um escalar não nulo.

(iii) A adição de qualquer múltiplo de uma linha a qualquer outra linha.

Define-se operações elementares sobre as colunas de uma matriz $A$, de maneira análoga.

Vejamos também a definição da adjunta de uma transformação linear:

Considere dois espaços vetoriais $V$ e $W$ sobre um corpo $F$ e uma transformação linear $T$ de $V$ em $W$. Então $T$ induz uma transformação linear de $W^{*}$ em $V^{*}$, duais de $W$ e $V$ respectivamente, como segue. Suponhamos que $g$ seja um funcional linear sobre $W$ e seja

$$
f(\alpha)=g(T \alpha)
$$

para cada $\alpha$ em $V$. Então a igualdade acima define uma função $f$ de $V$ em $F$, a saber, a composta de $T$, uma função de $V$ em $W$, com $g$, uma função de $W$ em $F$. Como $T$ e $g$ são 
ambas lineares, temos que $f$ também é linear, isto é, $f$ é um funcional linear sobre $V$. Assim $T$ nos fornece uma função $T^{t}$ que a cada funcional linear $g$ sobre $W$ faz corresponder um funcional linear $f=T^{t} g$ sobre $V$, definido na igualdade acima. Notemos também que $T^{t}$ é na verdade uma transformação linear de $W^{*}$ em $V^{*}$; de fato, se $g_{1}$ e $g_{2}$ estão em $W^{*}$ e $c$ é um escalar

$$
\left[T^{t}\left(c g_{1}+g_{2}\right)\right](\alpha)=\left(c g_{1}+g_{2}\right) T \alpha=c g_{1}(T \alpha)+g_{2}(T \alpha)=c\left(T^{t} g_{1}\right)(\alpha)+\left(T^{t} g_{2}\right)(\alpha)
$$

de modo que $T^{t}\left(c g_{1}+g_{2}\right)=c T^{t} g_{1}+T^{t} g_{2}$.

Teorema 1.1.1. Sejam $V$ e $W$ espaços vetoriais sobre um corpo $F$. Para cada transformação linear $T$ de $V$ em $W$ existe uma única transformação linear $T^{t}$ de $W^{*}$ em. $V^{*}$ tal que

$$
\left(T^{t} g\right)(\alpha)=g(T \alpha)
$$

para todo $g$ em. $W^{*}$ e $\alpha$ em $V$.

Denominamos $T^{t}$ como a adjunta (ou transposta) de $T$.

Outro fato a recordar é: se det è a função determinante das matrizes quadradas sobre um anel comutativo com unidade, e se $\alpha_{1}, \ldots, \alpha_{n}$ são as linhas da matriz $A$, escrevemos $\operatorname{det} A=\operatorname{det}\left(\alpha_{1}, \ldots, \alpha_{n}\right)$, então

$$
\operatorname{det}\left(\alpha_{1}, \ldots, c \alpha_{i}+\alpha_{i}^{\prime}, \ldots, \alpha_{n}\right)=c \operatorname{det}\left(\alpha_{1}, \ldots, \alpha_{i}, \ldots, \alpha_{n}\right)+\operatorname{det}\left(\alpha_{1}, \ldots, \alpha_{i}^{\prime}, \ldots, \alpha_{n}\right) .
$$

Esse mesmo resultado é válido se considerarmos $\alpha_{1}, \ldots, \alpha_{n}$ as colunas da matriz $A$.

Os resultados abaixo sobre grupos e anéis podem ser encontrados com maiores detalhes em Cohn [4].

Vejamos inicialmente que a interseç̧ão de qualquer conjunto de subgrupos de um grupo $G$ (mesmo um número infinito) é um subgrupo de $G$. Se $X$ é qualquer subconjunto de $G$ podemos considerar a coleção de todos subgrupos $H$ de $G$ tal que $X \subseteq H$ e formar suas intersecções, digamos $T$. Então $T$ será um subgrupo de $G$ e pode ser caracterizado como o menor subgrupo contendo $X$. Um modo mais construtivo para descrever $T$ seria: seja $T^{\prime}$ o conjunto de todos os produtos nos elementos de $X \cup X^{-1}$, isto é, os produtos formados pelos elementos de $X$ e seus inversos, incluindo 1 como o produto vazio. $T^{\prime}$ é um subgrupo e $T^{\prime} \supseteq X$. E mais, qualquer subgrupo contendo $X$ também contém $T^{\prime}$. Logo $T \supseteq T^{\prime}$, mas da definição de $T$ como a intersecção de todos os subgrupos contendo $X$ temos $T \subseteq T^{\prime}$, com isso $T=T^{\prime}$. Esse subgrupo $T$ é chamado o subgrupo gerado por $X$ e escrito $\langle X\rangle$, enquanto $X$ é chamado conjunto gerador de $T$.

Recordemos também da definição do produto direto de dois grupos: 
Definição 1.1.2. Sejam $G$ grupo e $A, B$ subgrupos de $G$; então $G$ é o produto direto de $A$ e $B$ se cada elemento de $A$ comuta com cada elemento de $B$ e $g$ de $G$ pode ser unicamente escrito na forma

$$
g=a b \quad(a \in A, b \in B)
$$

A definição pode também ser escrita como segue

Proposição 1.1.3. Um grupo $G$ é o produto direto de: seus subgrupos $A$ e $B$ se, e somente se, $A, B$ são normais em $G, G=A B$ e $A \cap B=1$.

Mais geralmente, chamamos um grupo $G$ o produto direto dos subgrupos $A_{1}, \ldots, A_{r}$ se quaisquer dois dos grupos $A_{i}$ comutam elemento a elemento e todo $g \in G$ pode ser unicamente escrito da forma

$$
g=a_{1} a_{2} \ldots a_{r} \quad\left(a_{i} \in A_{i}\right)
$$

Analogamente à proposição anterior, temos por indução

Corolário 1.1.4. Sejam $G$ grupo e $A_{1}, \ldots, A_{r}$ subgrupos quaisquer de $G$; então $G$ é o produto direto de $A_{1}, \ldots, A_{r}$ se, e somente se, cada $A_{i}$ é normal em $G, G=A_{1} A_{2} \ldots A_{r} e$ $A_{1} \ldots A_{i-1} \cap A_{i}=1$ para $i=2, \ldots, r$.

Para uma generalização do produto direto: sejam $A, B$ dois grupos e suponha que $A$ age por automorfismos sobre $B$. Isso significa que cada $a \in A$ define um automorfismo de $B$, escrito $b \mapsto b^{a}$, de tal modo que

$$
b^{1}=b, \quad\left(b^{a}\right)^{a^{\prime}}=b^{a a^{\prime}}, \quad\left(a, a^{\prime} \in A, b \in B\right) .
$$

Temos assim um homomorfismo $\lambda: A \rightarrow \operatorname{Aut} B$, e reciprocamente, qualquer homomorfismo $\lambda$ define uma $A$-ação sobre $B$.

Então podemos definir um grupo $G$, chamado o produto semi-direto de $A$ e $B$ com a ação dada de $A$ sobre $B$, como segue. Os elementos de $G$ são novamente pares $(a, b), a \in A, b \in B$, com multiplicação

$$
\left(a_{1}, b_{1}\right)\left(a_{2}, b_{2}\right)=\left(a_{1} a_{2}, b_{1}^{a_{2}} b_{2}\right)
$$

Essa multiplicação é associativa:

$$
\left[\left(a_{1}, b_{1}\right)\left(a_{2}, b_{2}\right)\right]\left(a_{3}, b_{3}\right)=\left(a_{1} a_{2} a_{3}, b_{1}^{a_{2} a_{3}} b_{2}^{a_{3}} b_{3}\right)=\left(a_{1}, b_{1}\right)\left[\left(a_{2}, b_{2}\right)\left(a_{3}, b_{3}\right)\right] ;
$$

o neutro é $\left(1_{A}, 1_{B}\right)$ e $(a, b)^{-1}=\left(a^{-1},\left(b^{-1}\right)^{a^{-1}}\right)$. Temos assim um grupo; os elementos $(a, 1)$ formam um subgrupo $A_{1}$ isomorfo a $A$ e os elementos $(1, b)$ formam um subgrupo $B_{1}$ isomorfo a $B$. $B_{1}$ é normal em $G$ e $G / B_{1} \cong A$; em geral $A_{1}$ não é normal em $G$. 
Recordemos que $R$ é um Domínio de Integridade se $R$ é um anel não trivial comutativo sem divisores de zero.

Seja $R$ um Domínio de Integridade e suponha que para cada $a \in R, a \neq 0$, um intciro não negativo $\varphi(a)$ está associado tal que

1. $\varphi(a b) \geq \varphi(a)$ para todo $a, b \neq 0$ em $R$,

2. para quaisquer $a, b \in R$, se $b \neq 0$, existe $q, r \in R$ tal que

$$
a=b q+r, \quad \text { oncls: } \varphi(r)<\varphi(b) \text { on } r=0 .
$$

Geralmente assumimos que $\varphi$ não é constante, isto i, $\varphi$ assune mais que un valor (por $2, \varphi$ pode ser constante apenas se $R$ é um corpo). Qualquer domínio com essas propriedades é dito ser Euclidiano. Em geral $\mathbb{Z}$ é um anel Euclidiano se considerarmos $\varphi(a)=|a|$. Para verificar que um anel é Euclidiano é mais conveniente trocarmos a condição 2 acima por outra condição equivalente:

2'. Para quaisquer $a, b \in R, b \neq 0$, se $\varphi(a) \geq \varphi(b)$, existe $c \in R$ tal que

$$
\varphi(a-b c)<\varphi(a) \text { our } a=b c
$$

Teorema 1.1.5. Em um anel Euclidiano $R$ quaisquer dois elementos tem um máximo divisor comum e um minimo múltiplo comum; se o máximo divisor comum de a e b é d, então existem $u, v \in R$ tal que $d=a u+b v$.

Também é interessante recordarmos alguns resultados sobre grupos abolianos livres:

A expressão de um elemento de um grupo em termos de um conjunto de geradores simplifica muito no caso do grupo ser abeliano. Assim se $A$ é um grupo abeliano, grado por $a_{1}, \ldots, a_{n}$, todo elemento $x \in A$ pode ser escrito na forma

$$
x=u_{1}^{\left(r_{1}\right.} \ldots u_{n}^{\alpha_{n}} \quad \text { onde. } \alpha_{1}, \ldots, \alpha_{n} \in \mathbb{Z}
$$

ou quando $A$ é um grupo aditivo,

$$
x=\alpha_{1} a_{1}+\ldots+\alpha_{n} a_{n}
$$

Vamos adotar a notação aditiva, então temos (2); cun geral não podomos afirmar çue os coeficientes $\alpha_{1}, \ldots, \alpha_{n}$ são unicamente determinados por $x$. Em geral, se $a_{11}$ t.en ordem finita $m_{1}$, podemos trocar $\alpha_{1}$ por um múltiplo de $m_{1}$ e ainda obtemos $x$. Um objetivo natural 
seria encontrar um conjunto de geradores $\left\{a_{1}, \ldots, a_{n}\right\}$ para $A$ tal (pue tocko elemento $x$ porle ser escrito de um único modo na forma (2), onde $\alpha_{i}$ está restrito a $0 \leq \alpha_{i}<m_{i}$ no caso de $a_{i}$ ter ordem finita $m_{i}$. Então $a_{1}, \ldots, a_{n}$ é chamada base de $A$. Escrevendo $A$ como soma direta de grupos cíclicos $A_{i}$ :

$$
A=A_{1} \oplus \ldots \oplus A_{n}
$$

Reciprocamente, se $A$ pode ser escrito conno uma soma direta de grupos cíclicos, isso nos leva a representação (2) para seus elennentos, a qual ó única se os $\alpha_{i}$ são sulicient,cunento pequenos sempre que $a_{i}$ tem ordem finita.

Proposição 1.1.6. Se A é um grupo abeliano qualquer, então o subconjunto t $A$ de elementos de ordem finita é um subgrupo.

O subgrupo tA é clamado subgrupo de torção de $A$, seus elementos são chanados elementos de torção de $A$, enquanto os elementos restantes de $A$ são livres de torção. $O$ grupo $A$ é chamado de grupo de torção se $t A=A$, e livre de torção se $t A=0$ ( $t A$ é um grupo de torção e $A / t A$ é livre de torção).

Um grupo de torção finitamente gerado deve ser finito. Se $A$ é gerado por $a_{1}, \ldots, a_{n}$ e $a_{i}$ tem ordem finita $m_{i}$, então todo elemento pode ser escrito na forma (2) com $0 \leq \alpha_{i}<m_{i}$, então existe no máximo $m_{1} m_{2} \ldots m_{n}$ diferentes elementos em $A$.

Para descrever os grupos livre de torção finitamente gerados temos

Definição 1.1.7. Um grupo abeliano $A$ é dito ser abeliano livre solve $e_{1}, \ldots, e_{n}$ se todo elemento de $A$ pode ser escrito unicamente da forma

$$
\lambda_{1} e_{1}+\ldots+\lambda_{n} e_{n} \quad \text { onde } \lambda_{1}, \ldots, \lambda_{n} \in \mathbb{Z}
$$

Em outras palavras, un grupo abeliano livre o $11 n$ grupo com uma base consistinclo do elementos livre de torção. É claro que $A$ terá em geral muitas bases diferentes, mas todas terão o mesmo número de elementos pois, escrevendo $2 A=\{2 x ; x \in A\}$, esse número é $n$, onde

$$
[A: 2 A]=2^{n} \text {. }
$$

Esse número $n$ é chamado o rank do grupo abeliano livre $A$.

Teorema 1.1.8. Seja $F_{n}$ um grupo abeliano livre sobre $E=\left\{e_{1}, \ldots, e_{n}\right\}$. Então qualquer aplicação de $E$ em um grupo abeliano $A$ estende a um único homomorfismo $F_{n} \rightarrow A$. Em particular, qualquer grupo abeliano finitamente gerado pode ser escrito como uma imagem. homomorfa de um grupo abeliano livre. 
Assim, $F_{n}$ com a aplicação inclusão $E \rightarrow F_{n}$ é universal para aplicações de $E$ em grupos abelianos livres.

A definição de um grupo abeliano livre pode ser estendida para o caso de rank infinito e todo grupo abeliano pode ser escrito como uma imagem homomorfa de um grupo abeliano livre adequado. Para grupos abelianos finitamente gerados, o grupo livre tem uma caracterização simples que não estende ao caso geral:

Proposição 1.1.9. Um grupo abeliano finitamente gerado é livre se, e somente se, é livre de torção.

Corolário 1.1.10. Todo grupo abeliano finitamente gerado é a soma direta de um grupo finito e um grupo livre.

\subsection{Homologia e cohomologia}

Podemos encontrar mais detalhes em Spanier [19]:

Definição 1.2.1. Um complexo de cadeias $C=\left(C_{q}, \partial_{q}\right)$ é definido por um par de seqüências, sendo uma seqüência de grupos abeiianos $C_{q}, q \in \mathbb{Z}$, e uma seqüência de homomorfismos $\partial_{q}: C_{q} \rightarrow C_{q-1}$, tal que $\partial_{q-1} \circ \partial_{q}=0$ para todo $q \in \mathbb{Z}$. É definido também $C_{q}=0$ se $q<0$.

A um complexo de cadeias $C=\left(C_{q}, \partial_{q}\right)$ se associa de modo natural certos subgrupos dos grupos de cadeias $C_{q}$. Os homomorfismos $\partial_{q}$ são denominados operadores bordo, os elementos de $C_{q}$ são chamados de $q$-cadeias, o grupo $Z_{q}(C)=k e r \partial_{q}$ é chamado de grupo dos $q$-ciclos, o grupo $B_{q}(C)=I m \partial_{q+1}$ é chamado de grupo dos $q$-bordos. Sendo $\partial_{q-1} \circ \partial_{q}=0$, então $B_{q}(C) \subset Z_{q}(C)$ e o grupo quociente

$$
H_{q}(C)=Z_{q}(C) / B_{q}(C)
$$

é chamado de $q$-ésimo grupo de homologia do complexo $C$.

Sejam $C$ um complexo de cadeias e $G$ um grupo abeliano. Podemos formar o complexo de cadeias $C \otimes G=\left(C_{q} \otimes G, \partial_{q} \otimes 1\right)$ com $\partial_{q} \otimes 1: C_{q} \otimes G \rightarrow C_{q-1} \otimes G$, onde $1: G \rightarrow G$ é o homomorfismo identidade. Definimos $H_{q}(C ; G)=H_{q}(C \otimes G)$ como sendo o $q$-ésimo grupo de homologia do complexo $C$ com coeficientes no grupo $G$.

A relação entre os grupos $H_{q}(C ; G)$ e $H_{q}(C)$ é dada pelo seguinte resultado:

Teorema 1.2.2. (Teorema dos Coeficientes Universais para Homologia) Sejam C um complexo de cadeias livre, isto é, os $C_{q}$ são grupos abelianos livres, e $G$ um grupo abeliano. Então

$$
H_{q}(C ; G) \cong H_{q}(C) \otimes G \oplus \operatorname{Tor}\left(H_{q-1}(C), G\right)
$$


Definição 1.2.3. Sejam $G$ um grupo abeliano e $C=\left(C_{q}, \partial_{q}\right)$ um complexo de cadcias. Formamos o grupo abeliano $C^{\|}=\operatorname{Hom}\left(C_{q}, G\right)$, isto é, seus elementos säo homomorfismos $h: C_{q} \rightarrow G$ chamados de q-cocadeias de $C$. O cobordo de he uma $(q+1)$-cocadeia, denotada por $\delta^{q} h, \delta^{q} h=(-1)^{q+1} h \circ \partial_{q+1}: C_{q+1} \rightarrow G$. Em outras, palavras o operador bordo $\partial_{q+1}: C_{q+1} \rightarrow C_{q}$ induz $\partial_{q+1}^{*}: \operatorname{Hom}\left(C_{q}, G\right) \rightarrow \operatorname{Hom}\left(C_{q+1}, G\right)$ e $\delta^{q}=(-1)^{q+1} \partial_{q+1}^{*}$. Observe que $\delta^{q} \circ \delta^{q-1}=0$. Seguc-se que $\left(C^{q}, \delta^{q}\right)$ é um complexo de cocadeias com cocficientes em G. Denotaremos Hom $(C, G)=\left(\operatorname{Hom}\left(C_{q}, G\right), \delta^{\eta}\right)$. A homologia do complexo $\operatorname{Hom}(C, G)$ é a. cohomologia de $C$ com coeficientes em $G$. Denotaremos por $H^{\prime \prime}(C ; G)$, istos a,

$$
H^{q}(C ; G)=\operatorname{ker} \delta^{q} / \operatorname{Im} \delta^{q-1} .
$$

Um elemento de $\operatorname{ker}\left(\delta^{q}\right)$ ó chamado de q-cociclo e um elemento de $\operatorname{Im}\left(\delta^{q-1}\right)$ é chamado de $q$-cobordo.

Teorema 1.2.4. (Coeficientes Universais para Cohomologia) Sejam C um complexo de. cadeias livre e $G$ um grupo abeliano. Então,

$$
H^{\prime \prime}(C ; G) \cong \operatorname{Hom}\left(H_{q}(C), G\right) \oplus \operatorname{Ext}\left(H_{\eta-1}(C), G\right) .
$$

Em Greenberg [8] podemos encontrar os resultados de homologia e cohomologia singular para um espaço topológico $X$, descritos abaixo.

Considere os vetores $E_{0}=(0,0, \ldots), E_{1}=(1,0, \ldots), E_{2}=(0,1,0, \ldots), \ldots$ em $\mathbb{R}^{\infty}$. Identificamos $\mathbb{R}^{n}$ como o subespaço onde todas as componentes depois da n-ésima são iguais a zero. Para qualquer $q \geq 0, \Delta_{q}$ é o simplexo geométrico $q$-dimensional gerado por $E_{0}, \ldots, E_{q}$, chamado $q$-simplexo padrão.

Sendo $P_{0}, \ldots, P_{q}$ pontos en algum espaço afim $E,\left(P_{0}, \ldots, P_{q)}\right)$ denotiará a restrição a $\Delta_{q}$ da única aplicação afim $\mathbb{R}^{\prime \prime} \rightarrow E$ aplicando $E_{0} \operatorname{ein} P_{0}, \ldots, E_{q} \operatorname{em} P_{q}$. Assim $\left(P_{0}, \ldots, P_{\eta \mid}\right)$ é a aplicação identidade de $\Delta_{\eta}$, denotada por $\delta_{q}$.

Dado um espaço $X$, um $q$-simplexo singular em $X$ é uma aplicaşão $\Delta_{q} \rightarrow X$. Definimos $S_{q}(X)$ como o $\mathbb{A}$-módulo livre gerado por todos os q-simplexos singulares (onde módulo é um grupo abeliano aditivo e $\mathbb{A}$ o ancl dos coeficientes). Os elementos de $S_{4}(X)$ são combinaçöes lineares

$$
\sum_{\sigma} v_{\sigma} \sigma
$$

onde $\sigma$ corresponde aos $q$-simplexos singulares, e os coeficientes $v_{\sigma}$ são de $\mathbb{A}$. Essas somas são chamadas q-cadeias singulares.

Para $q>0$, defina $F_{q}^{i}: \Delta_{q-1} \rightarrow \Delta_{q}$, para $0 \leq i<q$, sendo a apliciação afim $\left(E_{0} \ldots \widehat{E}_{i} \ldots E_{q}\right)$, onde $\widehat{E}_{i}$ significa omitir $E_{i}$. Com isso definimos a $i$-ésina face $\sigma^{(i)}$ de 
$\sigma$ como sendo o $(q-1)$-simplexo singular $\sigma \circ F_{q}^{i}$. Assim $F_{q}^{i}$ é a $i$-ésima face de $\delta_{q}$. $F_{q}^{i}$ aplica $\Delta_{q-1}$ afim e homeomorficamente sobre a face de $\Delta_{q}$ oposta ao vértice $E_{i}$.

Agora definimos o bordo de um $q$-simplexo singular sendo a $(q-1)$-cadeia singular

$$
\partial(\sigma)=\sum_{i=0}^{q}(-1)^{i} \sigma^{(i)}
$$

e $\partial \partial=0$.

Uma $q$-cadeia singular $c$ tal que $\partial(c)=0$ é chamada um ciclo; se $c=\partial\left(c^{\prime}\right)$ para alguma $(q+1)$-cadeia $c^{\prime}, c$ é chamada um bordo. Como $\partial \partial=0$ os bordos formam um submódulo $B_{q}$ do módulo $Z_{q}$ dos ciclos; e $Z_{q} / B_{q}$ é chamado o $q$-ésimo módulo de homologia singular de $X$, denotado por $H_{q}(X ; \mathbb{A})$, ou simplesmente $H_{q}(X)$ quando a referência ao anel $\mathbb{A}$ é conhecida.

Definição 1.2.5. O módulo $S^{q}(X)$ de todas as cocadeias singulares sobre $X$ é $H_{o m}\left(S_{q}(X), \mathbb{A}\right)=S_{q}(X)^{*}$. Assim a cocadeia singular de dimensão $q$ é um homomorfismo $\mathbb{A}$-linear $c: S_{q}(X) \rightarrow \mathbb{A}$. Se denotamos o valor desse homomorfismo sobre uma cadeia $z$ por $[z, c]$, temos então as igualdades

$$
\begin{gathered}
{\left[z_{1}+z_{2}, c\right]=\left[z_{1}, c\right]+\left[z_{2}, c\right]} \\
{\left[z, c_{1}+c_{2}\right]=\left[z, c_{1}\right]+\left[z, c_{2}\right]} \\
{[\nu z, c]=\nu[z, c]=[z, \nu c] \quad \nu \in \mathbb{A}}
\end{gathered}
$$

tal que [,] é bilinear.

Como $S_{q}$ é um funtor de espaços topológicos em $\mathbb{A}$-módulos e $H o m_{\mathbb{A}}(, \mathbb{A})$ é um contrafuntor na categoria de $\mathbb{A}$-módulos, o funtor composto $S^{q}$ é um contrafuntor de espaços topológicos em $\mathbb{A}$-módulos. Mais explicitamente, se $f: X \rightarrow Y$ é uma aplicação qualquer, então $S^{q}(f): S^{q}(Y) \rightarrow S^{q}(X)$ é definido pela fórmula

$$
\left[z, S^{q}(f) c\right]=\left[S_{q}(f) z, c\right]
$$

para qualquer $q$-cadeia $z, q$-cocadeia $c$. Quando $z$ é um $q$-simplexo singular $\sigma$, a fórmula se torna

$$
\left[\sigma, S^{q}(f) c\right]=[f \circ \sigma, c]
$$

em outras palavras, $S^{q}(f)$ é a transposta $S_{q}(f)^{t}$ de $S_{q}(f)$.

Proposição 1.2.6. Existe um único homomorfismo $\delta: S^{q}(X) \rightarrow S^{q+1}(X)$ satisfazendo

$$
[\partial z, c]=[z, \delta c]
$$


para toda $(q+1)$-cadeia $z$ e q-cocadeia $c$. Se $f: X \rightarrow Y$ é uma aplicação qualquer, então

$$
\delta S^{q}(f)=S^{q+1}(f) \delta
$$

E mais,

$$
\delta \delta=0
$$

Chamamos $\delta$ o operador cobordo.

Definimos os módulos de cociclos e cobordos por

$$
\begin{aligned}
& Z^{q}(X)=\text { Kernel } \delta: S^{q}(X) \rightarrow S^{q+1}(X) \\
& B^{q}(X)=\text { Imagem } \delta: S^{q-1}(X) \rightarrow S^{q}(X)
\end{aligned}
$$

e o grupo de cohomologia $H^{q}(X ; \mathbb{A})$ por

$$
H^{q}(X)=Z^{q}(X) / B^{q}(X) \text {. }
$$

Os grupos de homologia e cohomologia deste traballıo, terão coeficientes inteiros.

Seja

$$
S^{*}(X)=\oplus_{q>0} S^{q}(X)
$$

Vamos definir um produto, denominado cup, em $S^{*}(X)$ : queremos que seja bilinear e que $c \smile d \in S^{p+q}(X)$ se $c \in S^{p}(X)$ e $d \in S^{q}(X)$. Para definir $c \smile d$, é suficiente especificar $[\sigma, c \smile d]$ para qualquer $(p+q)$-simplexo singular $\sigma$. Para isso considere as aplicações afins

$$
\begin{aligned}
& \lambda_{p}: \Delta_{p} \rightarrow \Delta_{p+q} \\
& \rho_{q}: \Delta_{q} \rightarrow \Delta_{p+q}
\end{aligned}
$$

dadas por $\lambda_{p}=\left(E_{0} \ldots E_{p}\right), \rho_{q}=\left(E_{p} E_{p+1} \ldots E_{p+q}\right)$. Fazendo

$$
[\sigma, c \smile d]=\left[\sigma \lambda_{p}, c\right]\left[\sigma \rho_{\eta}, d\right]
$$

onde o lado direito é o produto de dois escalares em $\mathbb{A}$. Se $c=\Sigma_{p} c_{p}, d=\Sigma_{q} d_{q}$ são elementos arbitrários de $S^{*}(X)$, temos por definição

$$
c \smile d=\sum_{p, q} c_{p} \smile d_{q}
$$

Proposição 1.2.7. O produto cup em $S^{*}(X)$ é bilinear, associativo, e tem como elemento identidade a 0 -cocadeia 1 definida por $[x, 1]=1$ para todo ponto $x \in X$. 
Proposiçāo 1.2.8. O operador cobordo é uma derivação do anel-graduado $S^{*}(X)$, isto é,

$$
\delta(c \smile d)=\delta c \smile d+(-1)^{p} c \smile \delta d
$$

para $c \in S^{p}(X), d \in S^{q}(X)$.

Corolário 1.2.9. A soma direta $Z^{*}(X)$ do módulo dos cociclos é um subanel de $S^{*}(X)$ e a soma direta $B^{*}(X)$ do módulo dos cobordos é um ideal em $Z^{*}(X)$, conseqüentemente passando o produto cup ao quociente, a soma direta $H^{*}(X)$ dos módulos de cohomologia torna-se uma $\mathbb{A}$-álgebra graduada.

Seja $f: X \rightarrow Y$ uma aplicação contínua. Então $f$ induz homomorfismos $S^{q}(f): S^{q}(Y) \rightarrow$ $S^{q}(X)$ para todo $q$, e conseqüentemente um homomorfismo $S^{*}(f): S^{*}(Y) \rightarrow S^{*}(X)$ definido por

$$
S^{*}(f)\left(\sum_{p} c_{p}\right)=\sum_{p} S^{p}(f)\left(c_{p}\right)
$$

Similarmente temos um homomorfismo de módulo $H^{*}(f): H^{*}(Y) \rightarrow H^{*}(X)$.

Proposição 1.2.10. $S^{*}(f)$ e $H^{*}(f)$ são homomorfismos de anéis.

Corolário 1.2.11. $S^{*}$ e $H^{*}$ são contrafuntores da categoria de espaços topológicos para a categoria das $\mathbb{A}$-álgebras graduadas.

Teorema 1.2.12. $E m H^{*}(X)$ temos

$$
a \smile b=(-1)^{p q} b \smile a
$$

para $a \in H^{p}(X), b \in H^{q}(X)$. Em particular, se $a=b$ e p é impar, $a \smile a=0$, contanto que A tenha característica diferente de 2.

Ainda em Greenberg [8] temos

Teorema 1.2.13. (Fórmula de Künneth) Se $X$ e $Y$ são espaços topológicos, existe um isomorfismo natural

$$
H_{n}(X \times Y) \cong \sum_{p+q=n} H_{p}(X) \otimes H_{g}(Y) \oplus \sum_{r+s=n-1} \operatorname{Tor}\left(H_{r}(X), H_{s}(Y)\right)
$$

para todo $n$.

Em Spanier [19] podemos encontrar o seguinte resultado de homologia e cohomologia para variedades diferenciáveis: 
Teorema 1.2.14. (Dualidade de Poincaré) Seja $M$ uma n-variedade compacta com bordo $\partial M . S e(M, \partial M)$ é orientável, então:

$$
\begin{aligned}
& H^{\jmath}(M, \partial M) \cong H_{n-p}(M) \\
& H^{p}(M) \cong H_{n-p}(M, \partial M) .
\end{aligned}
$$

\subsection{Homotopia}

As seguintes definições podem ser encontradas em Hilton [10].

Sejam $X, Y$ espaço de Hausdorff conexos por caminhos e considere aplicações contínuas de $X$ em $Y$. Dizemos que $f_{0}, f_{1}: X \rightarrow Y$ são homotópicas se existe uma aplicação contínua $F: X \times I \rightarrow Y$, onde $I$ é o intervalo unitário $0 \leq t \leq 1$, tal que $F(x, 0)=f_{0}(x), F(x, 1)=$ $f_{1}(x)$ para todo $x \in X$ (se $f_{0}$ é homotópica a $f_{1}$ escrevemos $f_{0} \sim f_{1}$ ).

Agora sejam $f: X \rightarrow Y$ e $g: Y \rightarrow X$ tais que $g f: X \rightarrow X$ e $f g: Y \rightarrow Y$ sĩo homotópicas as aplicações identidades apropriadas. Então dizemos que $X$ e $Y$ tem o mesmo tipo de homotopia. Chamamos $f$ e $g$ equivalências de homotopia e $g(f)$ uma homotopia inversa de $f(g)$. Considerando 1 como a aplicação identidade de qualquer espaço nele mesmo, então $g f \sim 1$ e $f g \sim 1$.

Seja $I^{n}$ o $n$-cubo Euclidiano, consistinclo dos pontos $\left(t_{1}, \ldots, t_{n}\right)$ com $0 \leq t i \leq 1, i=$ $1 ; 2, \ldots, n$. O bordo de $I^{n}$, escrito $\dot{I}^{n}$, consiste dos pontos de $I^{n}$ onde $t_{i}=0$ para no mínimo um valor de $i$.

Agora seja $X$ um espaço de Hausdorff conexo por caminhos e seja $x_{0} \in X$. Sejam $f, g: I^{n} \rightarrow X$ tal que $f\left(1, t_{2}, \ldots, t_{n 2}\right)=g\left(0, t_{2}, \ldots, t_{n}\right)$ e defina $h: I^{n} \rightarrow X$ por

$$
h\left(t_{1}, \ldots, t_{n}\right)= \begin{cases}f\left(2 t_{1}, t_{2}, \ldots, t_{n}\right) & 0 \leq t_{1} \leq 1 / 2 \\ g\left(2 t_{1}-1, t_{2}, \ldots, t_{n}\right) & 1 / 2 \leq t_{1} \leq 1 .\end{cases}
$$

Então $h$ é contínua e escrevemos $h=f+g$.

Suponha agora que $f, y$ aplicam $I^{n}$ em $x_{0}$, c seja $M_{n}\left(X, x_{0}\right)$ a coleçño do todas as aplicações continuas de pares $\left(I^{\prime \prime}, \dot{I}^{n}\right) \rightarrow\left(X, x_{0}\right)$. Então $f+g$ está clctinida e pertence a $M_{n}\left(X, x_{0}\right)$. Assim temos uma adição em $M_{n}\left(X, x_{0}\right)$. Seja $\pi_{n}\left(X, x_{0}\right)$ a coleção de todas as classes de homotopia clas aplicações contínuas de pares $\left(I^{n}, I^{n}\right) \rightarrow\left(X, x_{1}\right)$, c seja $[f]$ in classe de $f$. Se $f, g \in M_{n}\left(X, x_{0}\right)$, então $[f+g]$ depende apenas de $[f]$ e $[g]$, e com isso a adição introduzida em $M_{n}\left(X, x_{0}\right)$ induz uma adição em $\pi_{n}\left(X, x_{0}\right)$.

A adição definida em $\pi_{n}\left(X, x_{0}\right)$ não é apenas descrita em termos da adição particular em $M_{n}\left(X, x_{0}\right)$, existem outras formas equivalentes de definir esta operação. 
Teorema 1.3.1. Segundo a operação de adição, a coleção de classes, $\pi_{n}\left(X, x_{0}\right)$, é um grupo. $\pi_{n}\left(X, x_{0}\right)$ é abeliano se $n>1$.

O grupo $\pi_{n}\left(X, x_{0}\right)$ é chamado o $n$-ésimo grupo de homotopia de $X$ com ponto base $x_{0} \in X$. Quando $n=1, \pi_{1}\left(X, x_{0}\right)$ é chamado de grupo fundamental de $X$.

Os próximos resultados podem ser encontrados em Spanier [19]:

Teorema 1.3.2. (Teorema de Hurewicz) Seja $X$ um espaço simplesmente conexo. Então, as seguintes afirmações são equivalentes:

$$
\begin{array}{ll}
\pi_{j}(X)=0, & 1 \leq j \leq n-1 \\
H_{j}(X)=0, & 1 \leq j \leq n-1
\end{array}
$$

No caso de uma das condições acima estar verificada, tem-se $H_{n}(X) \cong \pi_{n}(X)$.

Sejam $X$ e $Y$ espaços topológicos e $f: X \rightarrow Y$ uma aplicação contínua. Dizemos que $f$ é uma equivalência de homotopia fraca se $f$ induz uma correspondência biunívoca entre as componentes conexas por caminhos de $X$ e $Y$ e se para todo $q \geq 1$ e $x \in X$, $f_{\#}: \pi_{q}(X, x) \rightarrow \pi_{q}(Y, f(x))$ é um isomorfismo. Observe que se $f: X \rightarrow Y$ é uma equivalência de homotopia, então $f$ é uma equivalência de homotopia fraca. Se $X$ e $Y$ forem variedades de classe $C^{\infty}$, então tem a estrutura de $C W$-complexos e os conceitos de equivalência de homotopia fraca e equivalência de homotopia coincidem.

Também em Spanier [19], são estabelecidas correspondências entre os elennentos de $\pi_{n}\left(X, x_{0}\right)$ e as classes de homotopia das aplicações contínuas $f:\left(S^{n}, p_{0}\right) \rightarrow\left(X, x_{0}\right), p_{0} \in S^{n}$, ou $g:\left(D^{n}, S^{n-1}\right) \rightarrow\left(X, x_{0}\right)$, onde $D^{n}$ é o disco orientado $n$-dimensional unitário e $S^{n-1}$ sua fronteira, isto é, a $(n-1)$-esfera unitária.

Seja $Y$ um subespaço fechado e conexo por caminhos de $X$ e $y_{0} \in Y$. Os elementos do grupo de homotopia relativo $\pi_{n}\left(X, Y, y_{0}\right)$ estão em correspondência com as classeș de homotopia de aplicações contínuas $g^{\prime}:\left(D^{n}, S^{n-1}, p_{0}\right) \rightarrow\left(X, Y, y_{0}\right)$, onde $g^{\prime}\left(D^{n}\right) \subset X$, $g^{\prime}\left(S^{n-1}\right) \subset Y$ e $g^{\prime}\left(p_{0}\right)=y_{0}$. Note que um elemento de $\pi_{n-1}\left(Y, y_{0}\right)$ é representado pela restrição de $g^{\prime}$ a $S^{n-1},\left.g^{\prime}\right|_{S^{n-1}}:\left(S^{n-1}, p_{0}\right) \rightarrow\left(Y, y_{0}\right)$.

Considere $S^{p} \vee S^{q}=S^{p} \times\left\{q_{0}\right\} \cup\left\{p_{0}\right\} \times S^{q} \subset S^{p} \times S^{q}$, onde $p_{0} \in S^{p}, q_{0} \in S^{q}, p, q \geq 2$. Os elementos de $\pi_{p+q}\left(S^{p} \times S^{q}, S^{p} \vee S^{q}, *\right)$ estão em correspondência com as classes de homotopia de aplicações contínuas $g^{\prime}:\left(D^{p} \times D^{q}, \partial\left(D^{p} \times D^{q}\right), w\right) \rightarrow\left(S^{p} \times S^{q}, S^{p} \vee S^{q}, *\right)$, onde $g^{\prime}\left(D^{p} \times\right.$ $\left.D^{q}\right) \subset S^{p} \times S^{q}, g^{\prime}\left(\partial\left(D^{p} \times D^{q}\right)\right) \subset S^{p} \vee S^{q}$ e $g^{\prime}(w)=*$, com $w \in S^{p-1} \times S^{q-1}=\partial D^{p} \times \partial D^{q} \subset$ $\partial\left(D^{p} \times D^{q}\right)=S^{p+q-1} \mathrm{e} *=\left(p_{0}, q_{0}\right)$. Assim, cada elemento $\gamma \in \pi_{p+q}\left(S^{p} \times S^{q}, S^{p} \vee S^{q}, *\right)$ é representado por uma aplicação contínua $g^{t}:\left(D^{p} \times D^{q}, \partial\left(D^{p} \times D^{q}\right), w\right) \rightarrow\left(S^{p} \times S^{q}, S^{p} \vee S^{q}, *\right)$ : 
Observe que $H_{p+q}\left(S^{p} \times S^{\prime \prime}, S^{p} \vee S^{\prime \prime}\right) \cong \mathbb{Z}$. Segue-se clo teorema de Hurewicz relativo, cm Spanier [19] pag. 397, que $\pi_{p+q}\left(S^{p} \times S^{\prime \prime}, S^{\mu} \vee S^{\prime}, *\right) \cong H_{p+q}\left(S^{p} \times S^{\prime \prime}, S^{p} \vee S^{\prime}\right) \cong \mathbb{Z}$, pois $S^{p} \times S^{q}$ e $S^{p} \vee S^{q}$ são, neste caso, simplesmente conexos.

Defina o homomorfismo bordo

$$
d: \pi_{p+q}\left(S^{p} \times S^{q}, S^{p} \vee S^{\prime}, *\right) \rightarrow \pi_{p+q-1}\left(S^{p} \vee S^{q}, *\right)
$$

como sendo $d(\gamma)=\alpha_{1}$, onde $\alpha_{1}$ é a classe de homotopia representada por $\left.g^{\prime}\right|_{\partial\left(D^{p} \times D^{q}\right)}:\left(\partial\left(D^{p} \times D^{q}\right), w\right) \rightarrow\left(S^{p} \vee S^{q}, *\right)$.

Definição 1.3.3. (Colchete de Whitehead) considere as classes de homotopia $\alpha \in \pi_{p}\left(X, x_{0}\right) e$ $\beta \in \pi_{q}\left(X, x_{0}\right)$ representadas pelas aplicações continuas $g_{1}:\left(S^{p}, p_{0}\right) \rightarrow\left(X, x_{0}\right) e$ $g_{2}:\left(S^{q}, q_{0}\right) \rightarrow\left(X, x_{0}\right)$ e a aplicação $g_{1} \vee g_{2}:\left(S^{p} \vee S^{q}, *\right) \rightarrow\left(X, x_{0}\right) \operatorname{com}\left(g_{1} \vee g_{2}\right)\left(p_{0}, q_{0}\right)=$ $x_{0},\left(g_{1} \vee g_{2}\right)\left(x, q_{0}\right)=g_{1}(x),\left(g_{1} \vee g_{2}\right)\left(p_{0}, y\right)=g_{2}(y)$. Seja $\gamma$ um gerador de $\pi_{p+\eta}\left(S^{p} \times S^{\prime}\right.$, $\left.S^{p} \vee S^{q}, *\right) \cong \mathbb{Z}$ escolhido através da fixação de uma orientação adequada de $S^{p} \times S^{\prime \prime}$. Para. a composta abaixo

$$
\pi_{p+q}\left(S^{p} \times S^{q}, S^{p} \vee S^{q}, *\right) \stackrel{d}{\longrightarrow} \pi_{p+q-1}\left(S^{p} \vee S^{q}, *\right) \stackrel{\left(g_{1} \vee g_{2}\right)_{*}}{\longrightarrow} \pi_{p+q-1}\left(X, x_{0}\right)
$$

defina

$$
[\alpha, \beta]=\left(\left(g_{1} \vee g_{2}\right)_{\#} \circ d\right)(\gamma)=\left(g_{1} \vee g_{2}\right)_{\#}\left(\alpha_{1}\right) \in \pi_{p+q-1}\left(X, x_{0}\right)
$$

$O$ elemento $[\alpha, \beta]$ é chamado de Colchete de Whitehead (ou produto de Whitchead) de $\alpha$ e $\beta$. Observe que $[\alpha, \beta]$ só depende das classes de homotopia $\alpha$ e $\beta$ e não dos particulares representantes $g_{1}$ e $g_{2}$.

Una propriedade do Colchete de Whitehead que vamos precisar no segundo capítulo é a "bilinearidade", isto é, para todo $m, n \in \mathbb{Z}$ tem-se

$$
[m \alpha, n \beta]=m n[\alpha, \beta] .
$$

O fato de que o Colchete cle Whitehead $[\alpha, \beta]$ é bilinear cin $\alpha$ e $\beta$, se $p, q \geq 2$, pode ser encontrado em Whitehead [23].

Para os enunciados e demonstrações a seguir ver Cartan [3].

Proposição 1.3.4. A aplicação contínua $g_{1} \vee g_{2}: S^{p} \vee S^{q} \rightarrow X$ se estende a uma aplicação continua $F: S^{p} \times S^{q} \rightarrow X$ se, e somente se, $[\alpha, \beta]=0$ em $\pi_{p+q-1}\left(X, x_{0}\right)$. 
Definição 1.3.5. (Definição de $H$-espaço) Seja $\left(X, x_{0}\right)$ espaço topológico $X$ com ponto base $x_{0} \in X$. Dizemos que $X$ é um $H$-espaço se existe uma aplicação contínua $f: X \times X \rightarrow X$, com $f\left(x_{0}, x_{0}\right)=x_{0}$ tal que as aplicações $f_{1}: X \rightarrow X, f_{1}(x)=f\left(x, x_{0}\right)$, e $f_{2}: X \rightarrow X, f_{2}(x)=$ $f\left(x_{0}, x\right)$, são homotópicas à identidade id $: X \rightarrow X$. O ponto $x_{0} \in X$ é o elemento neutro de $X$ a menos de homotopia.

Foi demonstrado em Adams [1], que $S^{p}$ tem estrutura de $H$-espaço se, e somente se, $p=1,3,7$. Nestes casos, a aplicação $f: S^{p} \times S^{p} \rightarrow S^{p}$ é a multiplicação dos complexos, dos quatérnios e dos octônios de norma igual a 1 respectivamente (ver também Cartan [3]).

Proposição 1.3.6. Se $p$ é um número ímpar $p \neq 1,3,7$, ou seja, $S^{p}$ não é um $H$-espaço, então $[\gamma, \gamma]$ é um elemento de ordem 2 de $\pi_{2 p-1}\left(S^{p}\right)$, onde $\gamma$ é um gerador de $\pi_{p}\left(S^{p}\right) \cong \mathbb{Z}$.

\subsection{Topologia das variedades diferenciáveis}

Veremos agora algumas definições e propriedades sobre variedades diferenciáveis, que poderão ser vistas com maiores detalhes em Hirsch [11].

Seja $f: M \rightarrow N$ uma aplicação de classe $C^{1}$ (onde $M, N$ são variedades $C^{r}, r \geq 1$ ). Dizemos que $f$ é imersiva em $x \in M$ se a aplicação linear $T_{x} f: T_{x} M \rightarrow T_{f(x)} N$ for injetiva, e submersiva de $T_{x} f$ for sobrejetiva. Se $f$ é imersiva em todos os pontos de $M$ ela é uma imersão; se ela é submersiva em todo ponto, $f$ é uma submersão.

Dizemos que $f$ é um mergulho se $f$ é uma imersão que aplica $M$ homeomorficamente sobre sua imagem.

Sejam $M$ e $N$ variedades diferenciáveis orientadas de dimensão $n$, ambas sem bordo, com $M$ compacta e $N$ conexa. Seja $f: M \rightarrow N$ uma aplicação diferenciável de classe $C^{\infty}$ e . $x \in M$ um ponto regular de $f$ (ou seja, $x$ é um ponto onde $f$ é submersiva). Observe que, nestas condições, a derivada de $f$ no ponto $x$, denotada por $d_{x} f$, é um isomorfismo entre os espaços tangentes em $x$ e em $f(x)$. Dizemos que $f$ preserva a orientação em $x$ se $d_{x} f$ levà uma base de $T_{x} M$ da orientação de $M$ numa base de $T_{f(x)} N$ da orientação de $N$. Se isto não ocorre, dizemos que $f$ inverte a orientação em $x$.

Definição 1.4.1. (Grau de uma aplicação diferenciável) Seja $x \in M$ um ponto regular. Dizemos que o grau de $f$ no ponto $x$ é igual a 1 se $d_{x} f$ preserva a orientação. Denotaremos por $\operatorname{deg}_{x} f=1$. Se $d_{x} f$ inverte a orientação, então o grau de $f$ no ponto $x$ é igual $a-1$.

Para cada valor regular $y \in N$ de $f$, definimos também o grau de $f$ sobre $y$ como sendo

$$
\operatorname{deg}(f, y)=\sum_{x \in f^{-1}(y)} \operatorname{deg}_{x} f
$$


Se $f^{-1}(y)$ for vazio, $\operatorname{deg}(f, y)=0$ por definição. Observe que $f^{-1}(y)$ tem unn número finito de pontos, pois $M$ é compacta.

O grau de uma aplicação contínua $f$, denotado por degf, é definido como sendo o grau de uma aplicação $h, \operatorname{deg}(h, z)$, onde $h: M \rightarrow N$ é uma aplicação $C^{\infty}$ homotópica a $f$ e $z \in N$ é um valor regular para $h$.

Por resultados de Hirsch [11], tal aplicação $h: M \rightarrow N$ existe e degf independe de $h$ e $z$.

Se $N$ é compacta, então $f_{*}[M]=(\operatorname{deg} f)[N]$ em $H_{n}(N ; \mathbb{Z})$, com $[M]$ e $[N]$ classes fundamentais de $M$ eN respectivamente.

Agora, se $P, Q$ são variedades, uma isotopia de $P$ em $Q$ é uma homotopia

$$
\begin{aligned}
& F: P \times I \rightarrow Q, \\
& F(x, t)=F_{t}(x)
\end{aligned}
$$

tal que a aplicação

$$
\widehat{F}: P \times I \rightarrow Q \times I
$$

definida por $\widehat{F}(x, t)=\left(F_{t}(x), t\right)$, é um mergulho.

Quando $P=Q$ e cada $F_{t}$ é um difeomorfismo, e $F_{0}=1_{Q}$, então $F$ é charnada uma difeotopia.

Outro resultado de variedades diferenciáveis é o teorema de aproximação, o qual podemos encontrar em Haefliger [9], e diz

Teorema 1.4.2. (a) Qualquer mergulho topológico (respectivamente imersão topológica) de uma variedade diferenciável $V^{n}$ em uma variedade diferenciável $W^{m}$ pode ser aproximado por um mergulho diferenciável se $m \geq 3(n+1) / 2$ (resp. uma imersão diferenciável se $\dot{m}>3 n / 2$ ).

(b) Sejam $f_{0}, f_{1}$ dois mergulhos diferenciáveis (resp. imersões) de $V^{n}$ em $W^{m}$. Qualquer homotopia na categoria de mergulhos topológicos (resp. imersões) pode ser aproximado por uma isotopia diferenciável se $m>3(n+1) / 2$ (resp. uma homotopia na categoria de imersões diferenciáveis se $m>(3 n+1) / 2)$.

\subsection{Difeomorfismos do produtos de esferas}

As definições e os resultados a seguir podem ser encontrados em Lucas [15].

Denotaremos por * um ponto base de $S^{p}$, com uma orientação fixada e consideremos $\alpha_{i}$, com $i=1,2, \ldots, n$, classes de homologia que geram $H_{p}(M ; \mathbb{Z})$ representadas por $S^{p} \times\{*\} \times$ $\ldots \times\{*\},\{*\} \times S^{p} \times\{*\} \times \ldots \times\{*\}, \ldots,\{*\} \times \ldots \times\{*\} \times S^{p}$. 
Definição 1.5.1. Seja $f: M \rightarrow M$ um difeomorfismo e $f_{*}: H_{p}(M) \rightarrow H_{p}(M)$ dada por:

$$
f_{*}\left(\alpha_{j}\right)=\sum_{i=1}^{n} a_{i j} \alpha_{i}
$$

Chamamos a matriz

$$
I_{f}=\left(\begin{array}{cccc}
a_{11} & a_{12} & \ldots & a_{1 n} \\
a_{21} & a_{22} & \ldots & a_{2 n} \\
\vdots & \vdots & \ddots & \vdots \\
a_{n 1} & a_{n 2} & \ldots & a_{n n}
\end{array}\right)
$$

de matriz da induzida por $f$ na base $\left\{\alpha_{i}\right\}$ de $H_{p}(M ; \mathbb{Z})$.

Os $a_{i j}(i, j=1,2, \ldots, n)$ de cada coluna de $I_{f}$ são os graus das composições

$$
S^{p} \stackrel{k_{j}}{\longrightarrow} M \stackrel{f}{\longrightarrow} M \stackrel{\pi_{i}}{\longrightarrow} S^{p}
$$

onde $k_{j}$ é a inclusão do $j$-ésimo fator em $M$ e $\pi_{i}$ é a projeção no $i$-ésimo fator.

Vamos denotar $G L(n ; \mathbb{Z})=\{A \in M(n \times n ; \mathbb{Z}) ; \operatorname{det} A= \pm 1\}$, o grupo das matrizes de ordem $n$ em $\mathbb{Z}$ com determinante \pm 1 e munido da multiplicação.

Definição 1.5.2. Dizemos que uma matriz $A \in G L(n ; \mathbb{Z})$ é realizada por um difeomorfismo de $M$ se existe um difeomorfismo $f: M \rightarrow M$ tal que $I_{f}=A: H_{p}(M) \rightarrow H_{p}(M)$ na base canônica $\left\{\alpha_{i}\right\}$ de $H_{p}(M)$.

Definição 1.5.3. Seja $D_{p}$ o subgrupo de $G L(n ; \mathbb{Z})$ consistindo das matrizes $A \in G L(n ; \mathbb{Z})$ tais que $I_{f}=A$ para algum difeomorfismo $f: M \rightarrow M$.

Definição 1.5.4. Seja $\mathfrak{G}_{n}$ o grupo das permutações de ordem $n$, isto é, $\mathfrak{G}_{n}$ é o grupo das bijeções $\sigma:\{1,2, \ldots, n\} \rightarrow\{1,2, \ldots, n\}$. Para cada $\sigma \in \mathfrak{G}_{n}$, definimos $B_{\sigma}=\left(b_{i j}\right) \in$ $G L(n ; \mathbb{Z})$ como sendo

$$
b_{i j}= \begin{cases}1 & \text { se } i=\sigma(j) \\ 0 & \text { caso contrário. }\end{cases}
$$

Observemos que a aplicação contínua $\gamma: \mathfrak{G}_{n} \rightarrow G L(n ; \mathbb{Z})$ definida por $\gamma(\sigma)=B_{\sigma}$ é um monomorfismo. De fato, para $\sigma, \mu \in \mathfrak{G}_{n}$, considere $B_{\sigma}=\left(a_{i j}\right)$ e $B_{\mu}=\left(b_{j k}\right)$, onde

$$
a_{i j}=\left\{\begin{array}{ll}
1 & \text { se } \sigma(j)=i \\
0 & \text { caso contrário }
\end{array} \quad e \quad b_{j k}= \begin{cases}1 & \text { se } \mu(k)=j \\
0 & \text { caso contrário }\end{cases}\right.
$$

Faça $B_{\sigma} \cdot B_{\mu}=C=\left(c_{i k}\right)=\left(\sum_{j=1}^{n} a_{i j} b_{j k}\right)$. 
Seja $j=\mu(k)$. Então temos $c_{i k}=a_{i 1} b_{1 k}+a_{i 2} l_{2 k}+\cdots+a_{i n} b_{n k}=a_{i 1} \cdot 0+\ldots+a_{i j} \cdot 1+$ $\cdots+a_{i n} \cdot 0=a_{i j}$, ou seja

$$
\begin{gathered}
c_{i k}=1 \Leftrightarrow a_{i j}=1 \Leftrightarrow i=\sigma(j) \Leftrightarrow i=\sigma \circ \mu(k), \\
c_{i k}=0 \Leftrightarrow a_{i j}=0 \Leftrightarrow i \neq \sigma(j) \Leftrightarrow i \neq \sigma \circ \mu(k) .
\end{gathered}
$$

Isto implica que $C=B_{\sigma \circ \mu}=\gamma(\sigma \circ \mu)$ e tem-se que

$$
\gamma(\sigma) \cdot \gamma(\mu)=B_{\sigma} \cdot B_{\mu}=B_{\sigma \circ \mu}=\gamma(\sigma \circ / h)
$$

e logo $\gamma$ é um homomorfismo.

Mostremos que $\gamma$ é injetiva, isto é, se $\sigma \neq \mu$, então $\gamma(\sigma) \neq \sigma(\mu)$. Consiclere $\gamma(\sigma)=B_{\sigma}=$ $\left(a_{i j}\right)$ e $\gamma(\mu)=B_{\mu}=\left(b_{i j}\right)$. Como $\sigma \neq \mu$, existe $l$ com $1 \leq l \leq n$ tal que $\sigma(l) \neq \mu(l)$. Faça $m=\mu(l) \neq \sigma(l)=k$ e tem-se que

$$
\begin{array}{cccc}
\sigma(l)=k \Rightarrow a_{k l}=1 \quad \text { e } & a_{m l}=0, \\
\mu(l)=m \Rightarrow b_{m l}=1 \quad \text { e } & b_{k l}=0 .
\end{array}
$$

Logo $a_{k l} \neq b_{k l}$, ou seja, $B_{\sigma} \neq B_{\mu}$ e assim $\gamma(\sigma) \neq \gamma(\mu)$. Portanto $\gamma$ é injetiva.

De agora em diante, identificaremos $\mathfrak{G}_{n} \operatorname{com} \gamma\left(\mathfrak{G}_{n}\right) \subset G L(n ; \mathbb{Z})$ pelo isomorfismo $\gamma: \mathfrak{G}_{n} \rightarrow \gamma\left(\mathfrak{G}_{n}\right)$

Seja $\eta: G L(n ; \mathbb{Z}) \rightarrow G L\left(n ; \mathbb{Z}_{2}\right)$ o homomorfismo canônico, definido por $\eta(X)=\bar{X}$, onde $\dot{X}=\left(x_{i j}\right), \bar{X}=\left(\bar{x}_{i j}\right)$ e $\bar{x}_{i j} \equiv x_{i j}(\bmod 2)$, e seja $G_{1}=\eta^{-1}\left(\eta\left(\mathfrak{G}_{n}\right)\right)$. Uma matriz em $G L(n ; \mathbb{Z})$ está em $G_{1}$ se, e somente se, cada uma de suas linhas e colunas contém exatamente um impar.

Observe que $G_{1}$ é um subgrupo de $G L(n ; \mathbb{Z})$. Como identificamos $\mathfrak{G}_{n} \operatorname{com} \gamma\left(\mathfrak{G}_{n}\right)$, e $\gamma\left(\mathfrak{G}_{n}\right)$ é um subgrupo de $G L(n ; \mathbb{Z})$, pois é a imagem de um grupo por um homomorfismo, então temos que $\mathfrak{G}_{n}$ é um subgrupo de $G L(n ; \mathbb{Z})$. Agora, como cada matriz de $\mathfrak{G}_{n}$ possui entradas 0 ou 1 temos que $\eta\left(\mathfrak{G}_{n}\right)=\overline{\mathfrak{G}_{n}}$, e $\eta\left(\mathfrak{G}_{n}\right)$ é um subgrupo de $G L\left(n ; \mathbb{Z}_{2}\right)$.

Mostremos então que $G_{1}$ é um subgrupo de $G L(n ; \mathbb{Z})$ :

(i) $I \in G_{1}$ pois $\eta(I)=\bar{I}$ que é o elemento identidade do grupo $\eta\left(\mathfrak{G}_{n}\right)$.

(ii) Sejam $A, B \in G_{1}$, logo temos $\eta(A \cdot B)=\eta(A) \eta(B)$ que pertence a $\eta\left(\mathfrak{G}_{n}\right)$ pois este é grupo. Logo $A \cdot B \in G_{1}$.

(iii) seja $A \in G_{1}$, então $\eta(A) \in \eta\left(\mathfrak{G}_{n}\right)$ que é grupo, logo $[\eta(A)]^{-1}$ está em $\eta\left(\mathfrak{G}_{n}\right)$ e como $\eta$ é homomorfismo, temos $[\eta(A)]^{-1}=\eta\left(A^{-1}\right)$. Portanto, $A^{-1} \in G_{1}$, ou seja, $G_{1}$ é subgrupo de $G L(n ; \mathbb{Z})$. 
Além disso, seja $G_{2}$ o subgrupo de $G L(n ; \mathbb{Z})$ gerado por $\mathfrak{G}_{n}$ junto com a matriz diagonal $R \in G L(n ; \mathbb{Z})$ com entradas da diagonal $-1,1, \ldots, 1$, ou seja $G_{2}=\left\langle\mathfrak{G}_{n}, R\right\rangle$. Note que $G_{2}$ é um subgrupo de $G_{1}$, o que podemos ver diretamente da definição de subgrupo gerado.

Observação 1.5.5. $\eta$ é um epimorfismo. De fato, para $X, Y \in G L(n ; \mathbb{Z})$ temos que $\eta(X$. $Y)=\overline{X \cdot Y}=\bar{X} \cdot \bar{Y}=\eta(X) \cdot \eta(Y)$. Logo $\eta$ é um homomorfismo. Verifiquemos agora que $\eta$ é sobrejetiva: considere $X \in G L\left(n ; \mathbb{Z}_{2}\right)$ então as entradas de $X$ são $\overline{0}$ e $\overline{1}$, e o determinante de $X$ é diferente de $\overline{0}$. Basta então considerar a matriz $Y$ em $G L(n ; \mathbb{Z})$ com entradas 0 onde é $\overline{0}$ em $X$ e 1 onde é $\overline{1}$ em $X$. Assim, $\eta(Y)=X$ e portanto $\eta$ é sobrejetiva.

Além disso, o índice $\left[G L(n ; \mathbb{Z}): G_{1}\right]$ de $G_{1}$ em $G L(n ; \mathbb{Z})$ é igual a $2^{\left(n^{2}-n\right) / 2}\left(2^{1}-1\right)\left(2^{2}-\right.$ 1)...( $\left(2^{n}-1\right) / n$ !, por Newmam [17].

Observemos primeiro a ordem de $G L\left(n ; \mathbb{Z}_{2}\right)$ : seja $A \in G L\left(n ; \mathbb{Z}_{2}\right)$, então o determinante de $A$ é diferente de zero, ou seja, $A$ é não singular. Escrevamos $A$ como a matriz de seus vetores coluna, $A=\left[C_{1}, \ldots, C_{n}\right]$. Então, por $A$ ser não singular seus vetores coluna são linearmente independentes sobre $\mathbb{Z}_{2}$. Com isso vemos que existem $2^{n}-1$ escolhas possíveis para $C_{1}$, uma vez que apenas $C_{1}=0$ deve ser excluído. Suponha que escolhemos $C_{1}, C_{2}, \ldots, C_{k}$. Então $C_{k+1}$ pode ser escolhido como qualquer vetor o qual não fica no subespaço gerado pelos vetores independentes $C_{1}, \ldots, C_{k}$. Como esse subespaço contém $2^{k}$ elementos e como existem $2^{n}$ vetores no total, $C_{k+1}$ pode ser escolhido de $2^{n}-2^{k}$ maneiras. Disso segue que $A$ pode ser escolhido de $\left(2^{n}-1\right)\left(2^{n}-2\right) \ldots\left(2^{n}-2^{n-1}\right)$ maneiras. Logo, a ordem de $G L\left(n ; \mathbb{Z}_{2}\right)$ é

$$
\begin{gathered}
\left(2^{n}-1\right)\left(2^{n}-2\right) \ldots\left(2^{n}-2^{n-1}\right)=\left(2^{n}-1\right) 2\left(2^{n-1}-1\right) \ldots 2^{n-1}\left(2^{1}-1\right) \\
=2^{1+\ldots+n-1}\left(2^{1}-1\right)\left(2^{2}-1\right) \ldots\left(2^{n}-1\right)=2^{\left(n^{2}-n\right) / 2}\left(2^{1}-1\right)\left(2^{2}-1\right) \ldots\left(2^{n}-1\right) .
\end{gathered}
$$

No caso de $\eta\left(\mathfrak{G}_{n}\right)$, como $\mathfrak{G}_{n}$ é um grupo de permutação de $n$ elementos, então a ordem de $\mathfrak{G}_{n}$ é $n$ !. Assim, a ordem de $\eta\left(\mathfrak{G}_{n}\right)$ é $n$ !. Com isso temos

$$
\frac{\left|G L\left(n ; \mathbb{Z}_{2}\right)\right|}{\left|\eta\left(\mathfrak{G}_{n}\right)\right|}=\frac{2^{\left(n^{2}-n\right) / 2}\left(2^{1}-1\right)\left(2^{2}-1\right) \ldots\left(2^{n}-1\right)}{n !} .
$$

Mostremos agora que o índice $\left[G L(n ; \mathbb{Z}): G_{1}\right]$ de $G_{1}$ em $G L(n ; \mathbb{Z})$ é igual a $\left[G L\left(n ; \mathbb{Z}_{2}\right)\right.$ : $\left.\eta\left(\mathfrak{G}_{n}\right)\right]$.

Assim, por Lucas [15], temos: seja $m=\left[G L\left(n ; \mathbb{Z}_{2}\right): \eta\left(\mathfrak{G}_{n}\right)\right]$. Note que a ordem de $G L\left(n ; \mathbb{Z}_{2}\right)$ é finita e é igual a $2^{\left(n^{2}-n\right) / 2}\left(2^{1}-1\right)\left(2^{2}-1\right) \ldots\left(2^{n}-1\right)$. Em particular, $m$ é finito e é igual a

$$
\frac{2^{\left(n^{2}-n\right) / 2}\left(2^{1}-1\right)\left(2^{2}-1\right) \ldots\left(2^{n}-1\right)}{n !} .
$$


Consideremos as classes laterais à direita de $\mathfrak{G}=\eta\left(\mathfrak{G}_{n}\right)$ em $G L\left(n ; \mathbb{Z}_{2}\right)$ dadas por $\mathfrak{B} k_{1}, \mathfrak{G} k_{2}$, $\ldots, \mathfrak{G} k_{m}$, onde $k_{1}, k_{2}, \ldots, k_{m} \in G L\left(n ; \mathbb{Z}_{2}\right)$ são representantes de classes. Como $\eta$ é um epimorfismo existem elementos $g_{1}, g_{2}, \ldots, g_{m} \in G L(n ; \mathbb{Z})$ tais que $\eta\left(g_{i}\right)=k_{i}$. Afirmamos que $G_{1} g_{1}, G_{1} g_{2}, \ldots, G_{1} g_{m}$ são classes laterais à direita, distintas, de $G L(n ; \mathbb{Z})$. Pois se $G_{1} g_{i}=$ $G_{1} g_{j}$, então $g_{j} g_{i}^{-1} \in G_{1}$. Logo, $\eta\left(g_{j} g_{i}^{-1}\right)=\eta\left(g_{j}\right) \eta\left(g_{i}\right)^{-1}=k_{j} k_{i}^{-1} \in \mathfrak{G}=\eta\left(\mathfrak{G}_{n}\right)$ e segue-se que $\mathfrak{G} k_{i}=\mathfrak{G} k_{j}$ e $i=j$. Isto inostra que as classes laterais à direita $G_{1} g_{1}, G_{1} g_{2}, \ldots, G_{1} g_{m}$ sũo todas distintas.

Seja $g \in G L(n ; \mathbb{Z})$. Mostremos que $g \in G_{1} g_{i}$ para algum $g_{i}$. Tem-se que $\eta(g) \in G L\left(n ; \mathbb{Z}_{2}\right)$ e $\eta(g) \in \mathfrak{G} k_{i}$ para algum inteiro $i$, ou seja, $\eta(g)=\sigma k_{i}$ com $\sigma \in \mathfrak{G}=\eta\left(\mathfrak{G}_{n}\right)$. Considere $x=$ $g g_{i}^{-1}$. Tem-se $\eta(x)=\eta\left(g g_{i}^{-1}\right)=\eta(g) \eta\left(g_{i}\right)^{-1}=\sigma k_{i} k_{i}^{-1}=\sigma \in \mathfrak{G}$. Logo, $x \in \eta^{-1}\left(\eta\left(\mathfrak{G}_{n}\right)\right)=G_{1}$, ou seja, $g g_{i}^{-1} \in G_{1}$. Assim $g \in G_{1} g_{i}$. Segue-se que todo elemento de $G L(n ; \mathbb{Z})$ está ein alguma classe à direita de $G_{1}$ em $G L(n ; \mathbb{Z})$. Então, $G_{1} g_{1}, G_{1} g_{2}, \ldots, G_{1} g_{m}$ consistem de todas as classes à direita de $G_{1}$ em $G L(n ; \mathbb{Z})$. Portanto,

$$
\left[G L(n ; \mathbb{Z}): G_{1}\right]=m
$$

Outro inodo de verificarmos a igualdade entre os índices segue do isomorfismo abaixo

$$
\frac{G L(n ; \mathbb{Z})}{\eta^{-1}\left(\eta\left(\mathfrak{G}_{n}\right)\right)} \approx \frac{G L\left(n ; \mathbb{Z}_{2}\right)}{\eta\left(\mathfrak{G}_{n}\right)}
$$

ưma vez que $\eta$ é um epimorfismo.

Observação 1.5.6. O subgrupo $G_{2}$ é finito de ordem $2^{n} n$ !. De fato, $G_{2}$ é isomorfo ao produto semi-direto de $\left(\mathbb{Z}_{2}\right)^{n}$ por $\mathfrak{G}_{n}$ com respeito a ação padrão de $\mathfrak{G}_{n}$ sobre $\left(\mathbb{Z}_{2}\right)^{n}$.

Como $G_{2}=<\mathfrak{G}_{n}, R>$, sabemos que a ordem de $\mathfrak{G}_{n}=n$ ! e o produto de elementos de $\mathfrak{G}_{n}$ por $R$ apenas muda o elemento -1 de linha e coluna, e quando multiplicamos por mais fatores $R$ podemos fazer aparecer mais entradas -1. Logo, fixada uma matriz de $\mathfrak{G}_{n}$ podemos trocar alguns 1 por -1 e como as matrizes de $\mathfrak{G}_{n}$ possuem $n$ linhas, podemos fazer essa troca de $2^{n}$ maneiras diferentes, ou seja, para cada matriz fixada de $\mathfrak{G}_{n}$ podemos encontrar uma bijeção que troca 1 por -1 , entre as $n$ linlıas da matriz fixada e o conjunto das partes de $\{1,2, \ldots, n\}$. Logo, a ordem de $G_{2}$ é $2^{n} n !$. 


\section{Capítulo 2}

\section{Classificação das matrizes que representam automorfismos}

Todo difeomorfismo $f: M \rightarrow M$ induz um automorfismo $f_{*}$ de $H_{p}(M ; \mathbb{Z}) \cong \mathbb{Z}^{n}$, o qual pode ser considerado como um elemento de $G L(n ; \mathbb{Z})=\{A \in M(n \times n ; \mathbb{Z}) ; \operatorname{det} A= \pm 1\}$ e seja $D_{p}$ o subgrupo de $G L(n ; \mathbb{Z})$ consistindo de todos os automorfismos que são induzidos por difeomorfismos de $M$. O resultado principal deste capítulo é o Teorema 2.2.1, o qual nos diz que

(i) $D_{p}$ coincide com $G L(n ; \mathbb{Z})$ para $p=1,3,7$,

(ii) $D_{p}$ coincide com $G_{1}$ (o subgrupo de $G L(n ; \mathbb{Z}$ ), no qual cada elemento possui apenas um ímpar em cada linha e coluna), para $p$ ímpar com $p \neq 1,3,7$,

(iii) $D_{p}$ coincide com $G_{2}=\left\langle\mathfrak{G}_{n}, R>\right.$ (onde $\mathfrak{G}_{n}$ é o grupo das matrizes de permutação e $R$ é a matriz com entradas na diagonal $-1,1, \ldots, 1)$, para $p$ par.

Os lemas da seção 1 e o Teorema 2.2.1 da seção 2, podem ser encontrados em Lucas [14].

\subsection{Lemas básicos}

Nesta seção veremos alguns lemas necessários a demonstração do teorema de classificação das matrizes que representam automorfismos. O primeiro lema será usado para mostrarmos que $D_{p}$ contém os subgrupos $G L(n ; \mathbb{Z}), G_{1}, G_{2}$, dependendo de $p$. 
Lema 2.1.1. 1. O grupo $G L(n ; \mathbb{Z})$ é gerado pelas matrizes $\mathfrak{G}_{n}, R$ e a malriz

$$
T=\left(\begin{array}{ccccc}
1 & 1 & 0 & \ldots & 0 \\
0 & 1 & 0 & \ldots & 0 \\
0 & 0 & 1 & \ldots & 0 \\
\vdots & \vdots & \vdots & \ddots & \vdots \\
0 & 0 & 0 & \ldots & 1
\end{array}\right)
$$

2. O grupo $G_{1}$ é gerado pelas matrizes de $\mathfrak{G}_{n}, R$ c a matriz

$$
U=\left(\begin{array}{ccccc}
1 & 2 & 0 & \ldots & 0 \\
0 & 1 & 0 & \ldots & 0 \\
0 & 0 & 1 & \ldots & 0 \\
\vdots & \vdots & \vdots & \ddots & \vdots \\
0 & 0 & 0 & \ldots & 1
\end{array}\right)
$$

\section{Demonstraçāo:}

1. Se $X$ está no subgrupo gerado por todas as matrizes de $\mathfrak{G}_{n}, R$ e $T$, ou seja, $X \in$ $<\mathfrak{G}_{n}, R, T>$ então $\operatorname{det} X= \pm 1$ e $X \in G L(n ; \mathbb{Z})$.

Mostremos que para qualquer matriz $A \in G L(n ; \mathbb{Z})$, então $A \in\left\langle\mathfrak{G}_{n}, R, T\right\rangle$. Para isto, basta mostrar que $A$ pode ser transformada na matriz identidade $I_{n \times n}$ por um número finito de operações elementares-linha e elementares-coluna (ver definições dadas no capítulo 1), já que estas operações são representadas por multiplicações pelos elementos citados acina.

Isto será feito por indução sobre $n$.

Se $n=1$, então o resultado segue imediatamente.

Suponha $n \geq 2$, e considere a matriz $A$ como sendo

Para a última coluna de $A$, defina

$$
A=\left(\begin{array}{cccc}
a_{11} & a_{12} & \ldots & a_{1 n} \\
a_{21} & a_{22} & \ldots & a_{2 n} \\
\vdots & \vdots & \ddots & \vdots \\
a_{n 1} & a_{n 2} & \ldots & a_{n n}
\end{array}\right)
$$

$$
v=\min \left\{\left|a_{\text {in }}\right| ; 1 \leq i \leq n, a_{\text {in }} \neq 0\right\}
$$


Fazendo uma operação elementar-linha do tipo (i), vista no capítulo 1, se necessário, pode-se assumir que $v=\left|a_{n n}\right|$. Fazendo operações elementares-linha do tipo (iii $)$, vista no capítulo 1 , obtém-se

$$
\left(\begin{array}{ccccc}
a_{11}+v_{1} a_{n 1} & a_{12}+v_{1} a_{n 2} & \ldots & a_{1 n-1}+v_{1} a_{n n-1} & a_{1 n}+v_{1} a_{n n} \\
a_{21}+v_{2} a_{n 1} & a_{22}+v_{2} a_{n 2} & \ldots & a_{2 n-1}+v_{2} a_{n n-1} & a_{2 n}+v_{2} a_{n n} \\
\vdots & \vdots & \ddots & \vdots & \vdots \\
a_{n-11}+v_{n-1} a_{n 1} & a_{n-12}+v_{n-1} a_{n 2} & \ldots & a_{n-1 n-1}+v_{n-1} a_{n n-1} & a_{n-1 n}+v_{n-1} a_{n n} \\
a_{n 1} & a_{n 2} & \ldots & a_{n n-1} & a_{n n}
\end{array}\right)
$$

onde escolhemos $v_{i}, 1 \leq i \leq n-1$, tais que

(a) $v_{i}=0$ se $a_{\text {in }}=0$

(b) se $a_{i n} \neq 0$, então $\left|a_{i n}+v_{i} a_{n n}\right|<\left|a_{i n}\right|$.

Repetindo esta operação, pode-se obter uma matriz da seguinte forma:

$$
\left(\begin{array}{ccccc}
b_{11} & b_{12} & \ldots & b_{1 n-1} & 0 \\
b_{21} & b_{22} & \ldots & b_{2 n-1} & 0 \\
\vdots & \vdots & \ddots & \vdots & \vdots \\
b_{n-11} & b_{n-12} & \ldots & b_{n-1 n-1} & 0 \\
* & * & \ldots & * & b_{n n}
\end{array}\right)
$$

Seja

$$
B=\left(\begin{array}{cccc}
b_{11} & b_{12} & \ldots & b_{1 n-1} \\
b_{21} & b_{22} & \ldots & b_{2 n-1} \\
\vdots & \vdots & \ddots & \vdots \\
b_{n-11} & b_{n-12} & \ldots & b_{n-1 n-1}
\end{array}\right)
$$

Como $\operatorname{det} A= \pm 1$, segue que $b_{n n}= \pm 1$ e $\operatorname{det} B= \pm 1$, ou seja, $B \in G L(n-1 ; \mathbb{Z})$.

Aplicando uma operação elementar-linha do tipo (ii), vista no capítulo 1, se necessário, podemos assumir que $b_{n n}=1$ e por operações elementares-coluna, pode-se obter a matriz

$$
\left(\begin{array}{cc}
B & 0 \\
0 & 1
\end{array}\right)
$$

Pela hipótese de indução, obtém-se a matriz identidade $I_{n \times n}$ por operações elementares. Portanto $A \in<\mathfrak{G}_{n}, R, T>$ e $G L(n ; \mathbb{Z})=\left\langle\mathfrak{G}_{n}, R, T>\right.$. 
2. Denotemos os homomorfismos $\gamma, \eta$, ja definidos anteriormente, por $\gamma_{n}, y_{n}$, respectivamente. Recordemos que para $\eta_{n}: G L(n ; \mathbb{Z}) \rightarrow G L\left(n ; \mathbb{Z}_{2}\right)$ uma matriz $A \in G L(n ; \mathbb{Z})$ está em $G_{1}$ se, e somente se, $A$ contém em todas as linhas e colunas um único elemento impar e os outros todos pares. Também pela definição de $\gamma$, estamos identificando $\mathfrak{G}_{n}$ com um subgrupo de $G L(n ; \mathbb{Z})$ por $\gamma_{n}$ para todo $n \geq 2$.

Conıo $\eta_{n}(R), \eta_{n}(U) \in \eta_{n}\left(\mathfrak{G}_{n}\right)$, obviamente temos que $\left\langle\mathfrak{G}_{n}, R, U>\subset G_{1}\right.$. seja $A \in G_{1}$. Mostremos que $A \in\left\langle\mathfrak{G}_{n}, R, U\right\rangle$. Para isto, basta mostrar que $A$ pode ser transformada na matriz identidade $I_{n \times n}$ por um número finito cle operações elementiares, mas aqui, em lugar de operação do tipo (iii), temos que usar a operação do tipo a seguir:

(iii)' $\mathrm{A}$ adição de qualquer múltiplo par de uma linha a qualquer outra linha (similarmente para operaçōes elementares-coluna).

Vamos mostrar isso por indução sobre $n$.

Se $n=1$ é óbvio. Suponha que $n \geq 2$ e a matriz $A$ seja dada por

$$
A=\left(\begin{array}{cccc}
a_{11} & a_{12} & \ldots & a_{1 n} \\
a_{21} & a_{22} & \ldots & a_{2 n} \\
\vdots & \vdots & \ddots & \vdots \\
a_{n 1} & a_{n 2} & \ldots & a_{n n}
\end{array}\right)
$$

Defina

$$
v=\min \left\{\left|\cdot a_{i n}\right| ; 1 \leq i \leq n, a_{i n} \neq 0\right\}
$$

Por uma operação elementar-linlıa do tipo (i) pode-se assumir que $v=\left|a_{n n}\right|$. Por operacõos elementares-linha sobre a matriz $A$, obtém-se

$$
\left(\begin{array}{ccccc}
a_{11}+v_{1} a_{n 1} & a_{12}+v_{1} a_{n 2} & \ldots & a_{1 n-1}+v_{1} a_{n n-1} & a_{1 n}+v_{1} a_{n n} \\
a_{21}+v_{2} a_{n 1} & a_{22}+v_{2} a_{n 2} & \ldots & a_{2 n-1}+v_{2} a_{n n-1} & a_{2 n}+v_{2} a_{n n} \\
\vdots & \vdots & \ddots & \vdots & \vdots \\
a_{n-11}+v_{n-1} a_{n 1} & a_{n-12}+v_{n-1} a_{n 2} & \ldots & a_{n-1 n-1}+v_{n-1} a_{n n-1} & a_{n-1 n}+v_{n-1} a_{n n} \\
a_{n 1} & a_{n 2} & \ldots & a_{n n-1} & a_{n n}
\end{array}\right)
$$

onde $v_{i} \equiv 0(\bmod 2)$ e pode-se escolher $v_{i}, i=1,2, \ldots, n-1$, de tal modo que

(c) se $a_{i n}=0$, então $v_{i}=0$

(d) se $a_{i n} \neq 0$ e $\left|a_{i n}\right|>\left|a_{n n}\right|$, então $\left|a_{i n}+v_{i} a_{n n n}\right|<\left|a_{i n}\right|, v_{i} \equiv 0(\bmod 2)$

(e) se $a_{i n} \neq 0$ e $\left|a_{i n}\right|=\left|a_{n n}\right|$, então $v_{i}=0$. 
Se $a_{1 n}=a_{2 n}=\ldots=a_{n-1 n}=0, \operatorname{como} \operatorname{det} A= \pm 1$, então por' uma operação elementarlinha do tipo (ii) se necessário, podemos assumir que $a_{n n}=1$. Como $A \in G_{1}$ segue que $a_{n 1}, a_{n 2}, \ldots, a_{n n-1}$ são todos pares, em seguida, aplicando as operações elementarescoluna do tipo $(\text { iii })^{\prime}$ podemos assumir que $A$ é da forma

$$
\left(\begin{array}{ll}
C & 0 \\
0 & 1
\end{array}\right)
$$

para alguma matriz $C \in G L(n-1 ; \mathbb{Z})$.

Se não ocorrer a hipótese do parágrafo anterior, então podemos escolher $v_{i}, i=1,2, \ldots$, $n-1$, como em $(c),(d),(e)$. Neste caso, se $\left|a_{n n}\right|$ é um número par, pode ocorrer que para algum outro $\left|a_{k n}\right|$ tenhamos $\left|a_{k n}\right|=\left|a_{n n}\right|$ e assim, por $(e), v_{k}=0$. Mas nem todos os $v_{i}^{\prime} s$ são nulos, pois por hipótese $A \in G_{1}$, isto é, $A$ contém em todas as linhas e colunas um único elemento ímpar. Logo, para algum $i$, podemos aplicar $(d)$, ou seja, $\left|a_{i n}+v_{i} a_{n n}\right|<\left|a_{i n}\right|$. Seja $\left|a_{n n}\right|$ um número ímpar. Como por hipótese para algum $i, a_{i n} \neq 0$ e é par, segue que $\left|a_{i n}\right| \neq\left|a_{n n}\right|$. Portanto para algum $i, v_{i} \neq 0$ também. Repetindo este tipo de operação um número finito de vezes, podemos assumir que a hipótese do parágrafo anterior ocorre.

Mostremos que a matriz $C$ está em $\eta_{n-1}^{-1}\left(\eta_{n-1}\left(\mathfrak{G}_{n-1}\right)\right) \subset G L(n-1 ; \mathbb{Z})$. Como a matriz $A \in G_{1}$, isto significa que toda linha e toda coluna de $A$ contém apenas um elemento ímpar e os outros são todos pares. Então, para $C$ também apenas um elemento de cada linha e cada coluna é um número ímpar e os outros são pares. Logo $C \in \eta_{n-1}^{-1}\left(\eta_{n-1}\left(\mathfrak{G}_{n-1}\right)\right)$.

Aplicando a hipótese de indução, obtém-se a matriz identidade $I_{n \times n}$ por operações elementares dos tipos (i), (ii), (iii)'. Portanto $A \in\left\langle\mathfrak{G}_{n}, R, U\right\rangle$.

Os próximos dois lemas serão utilizados para demonstrar que $D_{p}$ está contido nos subgrupos $G L(n ; \mathbb{Z}), G_{1}, G_{2}$, dependendo de $p$.

Lema 2.1.2. Se $p$ é impar com $p \neq 1,3,7$, então para todo difeomorfismo $f: M \rightarrow M$, temos $I_{f} \in G_{1}$. 
Demonstração: Seja

$$
I_{f}=\left(\begin{array}{cccc}
a_{11} & a_{12} & \ldots & a_{1 n} \\
a_{21} & a_{22} & \ldots & a_{2 n} \\
\vdots & \vdots & \ddots & \vdots \\
a_{n 1} & a_{n 2} & \ldots & a_{n n}
\end{array}\right)
$$

realizada pelo difeomorfismo $f$, com determinante igual a \pm 1 .

Considere a seguinte composta

$$
S^{p} \times S^{p} \stackrel{k}{\longrightarrow} M \stackrel{f}{\longrightarrow} M \stackrel{\pi}{\longrightarrow} S^{p}
$$

onde $k$ e $\pi$ serão definidas a seguir. Sejam os geradores canônicos $w_{1}, w_{2} \in \pi_{p}\left(S^{p} \times S^{p}\right) \cong$ $\mathbb{Z} \oplus \mathbb{Z}, w \in \pi_{p}\left(S^{p}\right) \cong \mathbb{Z}$ e o homomorfismo abaixo:

$$
(\pi \circ f \circ k)_{\#}: \pi_{p}\left(S^{p} \times S^{p}\right) \rightarrow \pi_{p}\left(S^{p}\right)
$$

definido por

$$
\begin{aligned}
& (\pi \circ f \circ k)_{\#}\left(w_{1}\right)=a_{j i} w \\
& (\pi \circ f \circ k)_{\#}\left(w_{2}\right)=a_{j l} w
\end{aligned}
$$

onde $k$ é a inclusão para $i$ e $l$ ésimos fatores e $\pi$ é a projeção no $j$-ésimo fator. Tomemos a restrição de $\pi \circ f \circ k$ a $S^{p} \vee S^{p}=S^{p} \times\{v\} \cup\{u\} \times S^{p} \subset S^{p} \times S^{p}$, onde $u, v \in S^{p}$. Então pela definição de Colchete de Whitehead, a restrição $\left.\pi \circ f \circ k\right|_{S^{p} \vee S^{p}}$ determina um elemento de $\pi_{2 p-1}\left(S^{p}\right)$ que é o Colchete de Whitehead dado por:

$$
\left[a_{j i} w, a_{j l} w\right]=a_{j i} a_{j l}[w, w]
$$

pois o Colchete de Whitehead é bilinear. Como $S^{p}$ não é um $H$-espaço quando $p$ é ímpar com $p \neq 1,3,7$, então por Cartan [3] (prop. 3 e pag. 18) temos que $[w, w]$ é diferente de zéro e é um elemento de ordem 2. Como $\pi \circ f \circ k$ restrita a $S^{p} \vee S^{p}$ pode ser estendida a $S^{p} \times S^{p}$, então por Cartan [3] (prop. 2) obtemos

$$
a_{j i} a_{j l}[w, w]=0\left(\Leftrightarrow a_{j i} a_{j l} \equiv 0(\bmod 2)\right)
$$

Portanto, o produto de dois elementos quaisquer da mesma linha de $I_{f}$ é sempre um número par. Como $\operatorname{det} I_{f}= \pm 1$, cada linha e cada coluna da matriz $I_{f}$, tem exatamente um número impar, os demais são todos pares, isto implica que $\eta\left(I_{f}\right) \in \eta\left(\mathfrak{G}_{n}\right)$, ou seja, $I_{f} \in \eta^{-1}\left(\eta\left(\mathfrak{G}_{n}\right)\right)=G_{1}$. 
Lema 2.1.3. Se p ó par; culioo parn todo difcomorfismo $f: M \rightarrow M$ lemos $I_{f} \in Q_{2}$.

Demonstração: Sejam $I_{f}=\left(a_{i j}\right)$ e $\left\{\alpha_{i}^{*}\right\}$ a base de $H^{p}(M) \cong \operatorname{Hom}\left(H_{p}(M) ; \mathbb{Z}\right)$ clual a $\left\{\alpha_{i}\right\}$. Pelo Teorema da Dualidade de Poincaré, vemos que $\alpha_{1}^{*} \smile \alpha_{1}^{*}=\alpha_{2}^{*} \smile \alpha_{2}^{*}=\ldots=$ $\alpha_{n}^{*} \smile \alpha_{n}^{*}=0$, e que $\left\{\alpha_{i}^{*} \smile \alpha_{j}^{*}: 1 \leq i<j \leq n\right\}$ é uma base de $H^{2 p}(M) \cong H_{(n-2) p}(M) \cong$ $\mathbb{Z}^{\frac{n !}{(n-2)(21}}$. O homonorfisnı $f^{*}$ induzido por $f$ na cohomologia $H^{p}(M)$ corrcsponde a transposta $I_{f}^{t}$ de $I_{f}$ com respeito a base dual. Como $p$ é par, $\alpha_{i}^{*} \smile \alpha_{j}^{*}=\alpha_{j}^{*} \smile \alpha_{i}^{*}$ par'a $i, j=1, \ldots, n$. Assim, para todo $k \operatorname{com} 1 \leq k \leq n$, tomos $f^{*}\left(\alpha_{k}^{*}\right)=\sum_{i=1}^{n} a_{k i} \alpha_{i}^{*} \mathrm{c}$

$$
0=f^{*}(0)=f^{*}\left(\alpha_{k}^{*} \smile \alpha_{k}^{*}\right)=f^{*}\left(\alpha_{k}^{*}\right) \smile f^{*}\left(\alpha_{k}^{*}\right)=\sum_{1 \leq i<j \leq n} 2 u_{k i} a_{k j, j}\left(\alpha_{i}^{*} \smile \alpha_{j}^{*}\right) .
$$

Isso implica que $a_{k i} a_{k j}=0$ para $i \neq j$. Como $\operatorname{det} I_{f}= \pm 1$, temos que $I_{j} \in G_{2}$.

\subsection{Teorema de classificação das matrizes que represen- tam automorfismos}

Agora veremos uma generalização do caso estudado por Wall $[22]$ e Goldstein $[6]$ quando $n=2$.

Teorema 2.2.1. Temos

$$
D_{p}= \begin{cases}G L(n ; \mathbb{Z}) & \text { se } p=1,3,7 \\ G_{1} & \text { se } p \text { é impar e } p \neq 1,3,7 \\ G_{2} & \text { se p é par. }\end{cases}
$$

Demonstração: Para $\sigma \in \mathfrak{G}_{n}$, seja $f_{\sigma}: M \rightarrow M$ o difeomorfismo definido por

$$
f_{\sigma}\left(x_{1}, \ldots, x_{n}\right)=\left(x_{\sigma^{-1}(1)}, \ldots, x_{\sigma^{-1}(n)}\right),\left(x_{1}, \ldots, x_{n}\right) \in M
$$

A matriz de representação $I_{f_{\sigma}}$ de $\left(f_{\sigma}\right)_{*}: H_{p}(M) \rightarrow H_{p}(M)$ com respeito a basc $\left\{\alpha_{i}\right\}$ é clada por $\sigma \in \mathfrak{G}_{n} \subset G L(n ; \mathbb{Z})$. Scndo $\sigma=\left(a_{i j}\right)_{1 \leq i, j} \leq n$, podemos determinar $a_{n 1}$ atravís da composição

$$
S_{1} \stackrel{k_{1}}{\longrightarrow} M \stackrel{f_{\sigma}}{\longrightarrow} M \stackrel{\pi_{i}}{\longrightarrow} S^{p}
$$

onde $S_{1}=S^{p} \times\{*\} \times \ldots \times\{*\} \subset M$.

Para $i=1,2, \ldots, n$, temos

$$
\left(\pi_{i} \circ f_{\sigma} \circ k_{1}\right)(x, *, \ldots, *)= \begin{cases}x & \text { se } i=\sigma^{-1}(1) \\ * & \text { (aso contrário }\end{cases}
$$


Note que $a_{i 1}$ é igual ao grau de $\pi_{i} \circ f_{\sigma} \circ k_{1}$. Como $\operatorname{deg}\left(\pi_{i} \circ f_{\sigma} \circ k_{1}\right)$ é igual ao grau da aplicação constante para $i \neq \sigma^{-1}(1)$, temos $a_{i 1}=0$ para $i \neq \sigma^{-1}(1)$. Como $\operatorname{deg}\left(\pi_{\sigma^{-1}(1)} \circ f_{\sigma} \circ k_{1}\right)$ é igual ao grau da aplicação identidade, temos que $a_{\sigma^{-1}(1) 1}=1$.

Similarmente os graus de $\pi_{i} \circ f_{\sigma} \circ k_{j}$, da $j$-ésima coluna de $I_{f_{\sigma}}$ são $a_{\sigma^{-1}(j) j}=1$ e os demais $a_{i j}$ são todos nulos para $2 \leq j \leq n$.

Quando escrevemos na forma matricial obtemos $I_{f_{\sigma}}$.

Seja $r: S^{p} \rightarrow S^{p}$ a reflexão, definida por

$$
r\left(y_{1}, \ldots, y_{p+1}\right)=\left(-y_{1}, \ldots, y_{p+1}\right),\left(y_{1}, \ldots, y_{p+1}\right) \in S^{p}
$$

onde identificamos $S^{p}$ com a esfera unitária em $\mathbb{R}^{p+1}$. O grau de $r$ é igual a -1 . Assim, para o difeomorfismo $f_{r}: M \rightarrow M$, definido por

$$
f_{r}\left(x_{1}, \ldots, x_{n}\right)=\left(r\left(x_{1}\right), \ldots, x_{n}\right),\left(x_{1}, \ldots, x_{n}\right) \in M
$$

Se a matriz de representação $I_{f_{r}}$ é igual a $\left(a_{i j}\right)_{1 \leq i, j \leq n}$, podemos determinar $a_{i 1}$ através da composição

$$
S^{p} \stackrel{k_{1}}{\longrightarrow} M \stackrel{f_{r}}{\longrightarrow} M \stackrel{\pi_{i}}{\longrightarrow} S^{p}
$$

para $i=1, \ldots, n$. Temos que se $x=\left(y_{1}, \ldots, y_{p+1}\right) \in S^{p}$ então

$$
\begin{gathered}
\left(\pi_{1} \circ f_{r} \circ k_{1}\right)(x, *, \ldots, *)=r(x) \\
\left(\pi_{2} \circ f_{r} \circ k_{1}\right)(x, *, \ldots, *)=* \\
\vdots \\
\left(\pi_{n} \circ f_{r} \circ k_{1}\right)(x, *, \ldots, *)=*
\end{gathered}
$$

Observe que $a_{i 1}$ é igual ao grau de $\pi_{i} \circ f_{\sigma} \circ k_{1}$. Como $\operatorname{deg}\left(\pi_{i} \circ f_{\sigma} \circ k_{1}\right)$ é igual ao grau da aplicação constante para $i \neq 1$, temos $a_{i 1}=0$ para $i \neq 1$. Como $\operatorname{deg}\left(\pi_{1} \circ f_{\sigma} \circ k_{1}\right)=\operatorname{deg}(r)$, temos que $a_{11}=-1$.

Usando um raciocínio análogo, podemos mostrar que $a_{i i}=1$ para $i=2,3, \ldots, n$ e que $a_{i j}=0$ para $j \geq 2$ e $i \neq j$.

Note que a matriz de representação $I_{f_{r}}$ de $\left(f_{r}\right)_{*}$ é igual a $R$ dada no capítulo anterior.

Por Adams [1], temos que $S^{p}$ tem a estrutura de um $H$-espaço se, e somente se, $p=1,3,7$.

Assim, quando $p=1,3,7$, para $x_{1}, x_{2} \in S^{p}$, denotamos por $x_{1} x_{2}$ o produto de $x_{1}$ e $x_{2}$ induzido pela estrutura de $H$-espaço de $S^{p}$. Note que para todo $x \in S^{p}$, temos o inverso de " $x$ ", 
que denotaremos por $x^{-1}$. Definamos $f_{l}: M \rightarrow M$ por $f_{l}\left(x_{1}, \ldots, x_{n}\right)=\left(x_{1} x_{2}, x_{2}, \ldots, x_{n}\right)$. Observe que $f_{t}$ é um difeomorfismo cujo inverso é dado por

$$
f_{t}^{-1}\left(x_{1}, \ldots, x_{n}\right)=\left(x_{1} x_{2}^{-1}, x_{2}, \ldots, x_{n}\right)
$$

De fato, e para mostrarmos isso, provaremos que $\lambda: S^{p} \rightarrow S^{p}$ dada por $\lambda(x)=x^{-1}$ é uma aplicação $C^{\infty}$, quando $p=1,3,7$.

1. (Caso $p=1$ ) Mostremos que $\lambda: S^{1} \rightarrow S^{1}, \lambda(x)=x^{-1}$ á uma aplicação $C^{\infty}$. Vamıs identificar $\mathbb{R}^{2}$ com $\mathbb{C}$ (Complexos). Então os elementos da esfera $S^{1} \subset \mathbb{C}$ clada por

$$
S^{1}=\{z \in \mathbb{C} ;|z|=1\}
$$

são os complexos de módulo 1.

Sabemos que todo complexo não nulo $z \in \mathbb{C}$ possui inverso multiplicativo denotado por $z^{-1}$. É fácil verificar para $z \in S^{1}$ que $\bar{z}=z^{-1}$, onde $\bar{z}=a-l i$ é o conjugado do complexo $z=a+b i(a, b \in \mathbb{R})$. Assim a aplicação

$$
\lambda_{1}: \mathbb{R}^{2} \rightarrow \mathbb{R}^{2}, \lambda_{1}(a, b)=(a,-b)
$$

é $C^{\infty}$, cuja restrição

$$
\left.\lambda_{1}\right|_{S^{1}}: S^{1} \rightarrow S^{1}, \lambda_{1}(z)=z^{-1}=\bar{z}
$$

também é $C^{\infty}$, pois $S^{1}$ é uma subvariedade $C^{\infty}$ de $\mathbb{R}^{2}$.

2. (Caso $p=3$ ) Mostremos que $\lambda: S^{3} \rightarrow S^{3}, \lambda(x)=x^{-1}$ é $C^{\infty}$. Podemos identificar $\mathbb{R}^{4}$ com os quatérnios $\mathbb{H}$ por

$$
\left(x_{1}, x_{2}, x_{3}, x_{4}\right) \mapsto w=x_{1}+x_{2} i+x_{3} j+x_{4} k, w \in S^{3},
$$

o seu conjugado $\bar{w}=x_{1}-x_{2} i-x_{3} j-x_{4} k$ satisfaz $\bar{w}=w^{-1}$. Segue-se então que

$$
\lambda_{2}: \mathbb{R}^{4} \rightarrow \mathbb{R}^{4}
$$

definida por

$$
\lambda_{2}\left(x_{1}, x_{2}, x_{3}, x_{4}\right)=\left(x_{1},-x_{2},-x_{3},-x_{4}\right)
$$

é $C^{\infty}$, cuja restrição

$$
\left.\lambda_{2}\right|_{S^{3}}=\lambda: S^{3} \rightarrow S^{3}, \lambda(w)=w^{-1}=\bar{w}
$$

também é $C^{\infty}$, pois $S^{3}$ é uma subvariedade $C^{\infty}$ de $\mathbb{R}^{4}$. 
3. (Caso $p=7$ ) Mostremos que $\lambda: S^{7} \rightarrow S^{7}, \lambda(x)=x^{-1}$ é $C^{\infty}$.

o conjunto dos números octônios $\mathbb{O}$ (ou números de Cayley) é definido como

$$
\mathbb{O}=\left\{c=\left(w_{1}, w_{2}\right) ; w_{1}, w_{2} \in \mathbb{H}\right\}
$$

A multiplicação de dois octônios $c_{1}=\left(w_{1}, w_{2}\right)$ e $c_{2}=\left(w_{3}, w_{4}\right)$ é definida por

$$
c_{1} c_{2}=\left(w_{1}, w_{2}\right)\left(w_{3}, w_{4}\right)=\left(w_{1} w_{3}-\overline{w_{4}} w_{2}, w_{4} w_{1}+w_{2} \overline{w_{3}}\right)
$$

Os octônios tem um elemento neutro $e=(1,0)$ que satisfaz

$$
e c=c e=c
$$

para todo $c \in \mathbb{O}$. Também é natural identificar $e=(1,0) \in \mathbb{O}$ com o elemento $1 \in \mathbb{R} \subset \mathbb{O}$. Definimos o conjugado $\bar{c}$ de um octônio $c=\left(w_{1}, w_{2}\right)$ como sendo

$$
\bar{c}=\left(\overline{w_{1}},-w_{2}\right)
$$

Então $c \bar{c}=|c|^{2} e=\left(\left|w_{1}\right|^{2}+\left|w_{2}\right|^{2}\right) e$ pode ser identificado com o número real não negativo $|c|^{2}, \mathrm{e}|c|^{2}=0$ se, e somente se, $c=(0,0)$.

Se $c \neq(0,0)$, então o inverso de $c$, denotado por $c^{-1}$, é dado por

$$
c^{-1}=\bar{c} /|c|^{2}
$$

Observe que nos octônios $\mathbb{O}$ a multiplicação é distributiva com relação a adição e que forma uma álgebra de divisão, isto é, valem todos os axiomas de um corpo com exceções da comutatividade e associatividade. Como fizemos no caso anterior, os octônios podem se identificados com o $\mathbb{R}^{8}$ e os elementos da esfera $S^{7} \subset \mathbb{R}^{8}$ dada por

$$
S^{7}=\left\{c \in \mathbb{O} ;|c|^{2}=1\right\}
$$

são os octônios de módulo 1 , então $c^{-1}=\bar{c}$ para $c \in S^{7}$. Segue-se de modo similar ao anterior que $\lambda: S^{7} \rightarrow S^{7}, \lambda(x)=x^{-1}$ é $C^{\infty}$.

Com isso podemos concluir que $f_{t}^{-1}: M \rightarrow M$ é de classe $C^{\infty}$, ou seja, $f_{t}: M \rightarrow M$ definida por $f_{t}\left(x_{1}, \ldots, x_{n}\right)=\left(x_{1} x_{2}, x_{2}, \ldots, x_{n}\right)$ é um difeomorfismo.

Ainda para $p=1,3,7$, considere a composição abaixo 
onde $S_{1}=S^{p} \times\{*\} \times \ldots \times\{*\} \subset M$.

Seja $f: S^{p} \times S^{p} \rightarrow S^{p}$ a multiplicação dos complexos se $p=1$, dos quatérnios se $p=3$ c dos octônios se $p=7$, de norma igual a 1 , e vamos assumir que $*=x_{0}$ é o elemento neutro de $S^{p}$. Note que $f_{1}, f_{2}: S^{p} \rightarrow S^{p}$ definidas por $f_{1}(x)=f\left(x, x_{0}\right)$ e $f_{2}(x)=f\left(x_{0}, x\right)$ respectivamente são aplicações de grau 1, pois são homotópicas a aplicação identidacle. Considere as aplicações compostas abaixo

$$
\begin{gathered}
\left(\pi_{1} \circ f_{t} \circ k_{1}\right)\left(x_{1}, *, \ldots, *\right)=x_{1} *=x_{1}=f_{1}\left(x_{1}\right) \\
\left(\pi_{2} \circ f_{t} \circ k_{1}\right)\left(x_{1}, *, \ldots, *\right)=* \\
\vdots \\
\left(\pi_{n} \circ f_{t} \circ k_{1}\right)\left(x_{1}, *, \ldots, *\right)=*
\end{gathered}
$$

Assim $\operatorname{deg}\left(\pi_{1} \circ f_{t} \circ k_{1}\right)=\operatorname{deg}\left(f_{1}\right)=1$ e $a_{11}=1$. Os graus de $\left(\pi_{i} \circ f_{l} \circ k_{1}\right)$, para $i=2,3, \ldots, n$, são iguais a zero, portanto $a_{i 1}=0$ para $i \geq 2$.

Considere agora, as aplicações compostas abaixo

$$
S_{2} \stackrel{k_{2}}{\longrightarrow} M \stackrel{f_{t}}{\longrightarrow} M \stackrel{\pi_{i}}{\longrightarrow} S^{p}
$$

onde $S_{2}=\{*\} \times S^{p} \times\{*\} \times \ldots \times\{*\} \subset M$, e

$$
\begin{gathered}
\left(\pi_{1} \circ f_{l} \circ k_{2}\right)\left(*, x_{2}, *, \ldots, *\right)=* x_{2}=x_{2}=f_{2}\left(x_{2}\right) \\
\left(\pi_{2} \circ f_{t} \circ k_{2}\right)\left(*, x_{2}, *, \ldots, *\right)=x_{2} \\
\left(\pi_{3} \circ f_{t} \circ k_{2}\right)\left(*, x_{2}, *, \ldots, *\right)=* \\
\vdots \\
\left(\pi_{n} \circ f_{t} \circ k_{2}\right)\left(*, x_{2}, *, \ldots, *\right)=*
\end{gathered}
$$

Então, $\operatorname{deg}\left(\pi_{1} \circ f_{t} \circ k_{2}\right)=\operatorname{deg}\left(f_{2}\right)=1$. Portanto, $a_{12}=1$. Similarmente obtém-se $a_{22}=1$, $a_{i 2}=0$ para $i \geq 3$. Com um raciocínio análogo, temos $a_{i i}=1$ para $i \geq 3$ e $a_{i j}=0$ para $j \geq 3$ e $i \neq j$.

Com isso, vemos que para $p=1,3,7$, a matriz de representação de $\left(f_{l}\right)_{*}$ é dadá por

$$
T=\left(\begin{array}{ll}
1 & 1 \\
0 & 1
\end{array}\right) \oplus I_{n-2}
$$

onde $I_{n-2}$ denota a matriz identidade $(n-2) \times(n-2)$. 
Assim $T$ é da forma

$$
\left(\begin{array}{ccccc}
1 & 1 & 0 & \ldots & 0 \\
0 & 1 & 0 & \ldots & 0 \\
0 & 0 & 1 & \ldots & 0 \\
\vdots & \vdots & \vdots & \ddots & \vdots \\
0 & 0 & 0 & \ldots & 1
\end{array}\right)
$$

Agora, para cada $x \in S^{p}$ definamos as aplicações $C^{\infty}$,

$$
\theta_{x}: S^{p} \rightarrow S^{p} \text { e } \varphi_{x}: S^{p} \rightarrow S^{p}
$$

por

$$
\dot{\theta}_{x}(y)=y-2<x, y>x \text { e } \varphi_{x}(y)=x-2<x, y>y,
$$

para $y \in S^{p}$, respectivamente, onde $<,>$ denota o produto interno de $\mathbb{R}^{p+1}$. A aplicação $\theta_{x}$ é a reflexão com respeito ao hiperplano $H$ ortogonal a $x$.

Vamos verificar os graus destas aplicaçōes: $\operatorname{como} \theta_{x}$ é uma reflexão, o grau de $\theta_{x}$ é igual a -1. Para determinar o grau de $\varphi_{x}$, primeiramente observemos que para todo $x, x^{\prime}$, pontos de $S^{p}, \varphi_{x}$ e $\varphi_{x^{\prime}}$ são homotópicas, pois $S^{p}$ é conexa por caminhos. Como o grau de uma aplicação é um invariante por homotopia, vamos calcular o grau de $\varphi_{x}$ no ponto $x=(0, \ldots, 0,1) \in S^{p}$. Neste caso, para $y=\left(y_{1}, \ldots, y_{p+1}\right) \in S^{p}$, tem-se

$$
\begin{gathered}
\varphi_{x}(y)=(0, \ldots, 0,1)-2 y_{p+1}\left(y_{1}, \ldots, y_{p+1}\right) \\
=\left(-2 y_{p+1} y_{1}, \ldots,-2 y_{p+1} y_{p}, 1-2 y_{p+1}^{2}\right) .
\end{gathered}
$$

Seja $q=(0, \ldots, 0,-1) \in S^{p}$, então $\varphi_{x}(y)=q$ se, e somente se,

$$
\left(-2 y_{p+1} y_{1}, \ldots,-2 y_{p+1} y_{p}, 1-2 y_{p+1}^{2}\right)=(0, \ldots, 0,-1)
$$

Isto implica que $\varphi_{x}^{-1}(q)=\{(0, \ldots, 0,+1),(0, \ldots, 0,-1)\}$. Denotemos

$$
q_{+}=(0, \ldots, 0,+1) \text { e } q_{-}=(0, \ldots, 0,-1)
$$

Para calcular $d_{q_{+}} \varphi_{x}$ e $d_{q_{-}} \varphi_{x}$, vamos especificar claramente os espaços tangentes $T_{q_{+}} S^{p}$ e $T_{\varphi_{x}\left(q_{+}\right)} S^{p}=T_{q} S^{p}=T_{q_{-}} S^{p}$. Como sabemos o espaço tangente a $S^{p}$ num ponto $u \in S^{p}, T_{u} S^{p}$, consiste de vetores normais a $u$ no ponto $u$. Então $T_{q_{+}} S^{p}$ é o espaço $p$-dimensional, cuja base é

$$
\left\{\partial / \partial y_{1}, \ldots, \partial / \partial y_{p}\right\}
$$


Aqui, estamos denotando $\left(y_{1}, \ldots, y_{p+1}\right)$ como coordcnadas de $\mathbb{R}^{p+1}$. Vamos fixar a orientação de $S^{p}$ por esta base ordenada. Então, a base orientada de $T_{q} S^{p}=T_{q_{-}} S^{p}$ é

$$
\left\{\partial / \partial y_{1}, \ldots, \partial / \partial y_{p-1},-\partial / \partial y_{p}\right\}
$$

Agora vamos determinar a matriz $d_{q_{+}} \varphi_{x}$ em relação as bases acima.

Usando a equação

$$
\varphi_{: x}(y)=\left(-2 y_{p+1} y_{1}, \ldots,-2 y_{p+1} y_{p}, 1-2 y_{p+1}^{2}\right)
$$

tem-se

$$
\begin{gathered}
d_{q_{+}} \varphi_{x:}\left(\partial / \partial y_{1}\right)=\left.\left(-2 y_{p^{p+1}}, 0, \ldots, 0\right)\right|_{\left.\right|_{y=\eta_{+}}}=(-2,0, \ldots, 0)=-2\left(\partial / \partial y_{1}\right) \\
\vdots \\
d_{q_{+}} \varphi_{x}\left(\partial / \partial y_{p-1}\right)=\left.\left(0, \ldots, 0,-2 y_{p+1}, 0,0\right)\right|_{y=q_{+}}=(0, \ldots, 0,-2,0,0)=-2\left(\partial / \partial y_{p-1}\right) .
\end{gathered}
$$

Finalmente calculamos

$$
d_{q_{+}} \varphi_{x}\left(\partial / \partial y_{p}\right)=\left.\left(0, \ldots, 0,-2 y_{p+1} ; 0\right)\right|_{y=q_{+}}=(0, \ldots, 0,-2,0)=-2\left(\partial / \partial y_{p}\right)=2\left(-\partial / \partial y_{p}\right)
$$

$\mathrm{Na}$ forma matricial, podemos escrever $d_{q_{+}} \varphi_{x}$ como matriz de ordem $p$

$$
\left(\begin{array}{ccccc}
-2 & 0 & \ldots & 0 & 0 \\
0 & -2 & \ldots & 0 & 0 \\
\vdots & \vdots & \ddots & \vdots & \vdots \\
0 & 0 & \ldots & -2 & 0 \\
0 & 0 & \ldots & 0 & 2
\end{array}\right)
$$

Com isso concluímos que $q_{+}$é ponto regular e $\operatorname{deg}_{q_{+}}\left(\varphi_{x}\right)=(-1)^{p-1}=(-1)^{p+1}$.

Com um argumento similar mostra-se que a matriz de $d_{q_{-}}\left(\varphi_{x}\right)$ é da forma

$$
\left(\begin{array}{cccc}
2 & 0 & \ldots & 0 \\
0 & 2 & \ldots & 0 \\
\vdots & \vdots & \ddots & \vdots \\
0 & 0 & \ldots & 2
\end{array}\right)
$$

Consequentemente, $q_{-}$também é um ponto regular e $\operatorname{deg}_{q_{-}}\left(\varphi_{x}\right)=1$.

Logo $q$ é um valor regular e o grau de $\varphi_{x}$ é igual a $(-1)^{p+1}+1$.

Portanto as aplicações $\theta_{x}: S^{p} \rightarrow S^{p}$ e $\varphi_{x}: S^{p} \rightarrow S^{p}$ tem graus -1 e $(-1)^{p+1}+1$, respectivamente. 
Se $p$ é ímpar, então para o difeomorfismo $f_{u}: M \rightarrow M$ definido por

$$
f_{u}\left(x_{1}, \ldots, x_{n}\right)=\left(\theta_{x_{2}}\left(x_{1}\right), x_{2}, \ldots, x_{n}\right),\left(x_{1}, \ldots, x_{n}\right) \in M
$$

a matriz de representação de $\left(f_{u}\right)_{*}$ é dada por $I_{f_{u}}=U R$, onde

$$
U=\left(\begin{array}{ll}
1 & 2 \\
0 & 1
\end{array}\right) \oplus I_{n-2}
$$

ou seja,

$$
U R=\left(\begin{array}{ccccc}
-1 & 2 & 0 & \ldots & 0 \\
0 & 1 & 0 & \ldots & 0 \\
0 & 0 & 1 & \ldots & 0 \\
\vdots & \vdots & \vdots & \ddots & \vdots \\
0 & 0 & 0 & \ldots & 1
\end{array}\right)
$$

De fato, para isso considere a composta abaixo

$$
S_{1} \stackrel{k_{1}}{\longrightarrow} M \stackrel{f_{u}}{\longrightarrow} M \stackrel{\pi_{i}}{\longrightarrow} S^{p}
$$

onde $S_{1}=S^{p} \times\{*\} \times \ldots \times\{*\} \subset M$

Logo, para $i=1,2, \ldots, n$

$$
\begin{gathered}
\left(\pi_{1} \circ f_{u} \circ k_{1}\right)(x, *, \ldots, *)=\theta_{*}(x) \\
\left(\pi_{2} \circ f_{u} \circ k_{1}\right)(x, *, \ldots, *)=* \\
\vdots \\
\left(\pi_{n} \circ f_{u} \circ k_{1}\right)(x, *, \ldots, *)=*
\end{gathered}
$$

Assim, $\operatorname{deg}\left(\pi_{1} \circ f_{u} \circ k_{1}\right)=\operatorname{deg}\left(\theta_{x_{2}}\right)=-1$ e $a_{11}=-1$, e os graus de $\left(\pi_{i} \circ f_{u} \circ k_{1}\right)$ para $i \geq 2$ são iguais a zero, $\operatorname{logo} a_{i 1}=0$ para $i \geq 2$.

Considere agora a composição

$$
S_{2} \stackrel{k_{2}}{\longrightarrow} M \stackrel{f_{u}}{\longrightarrow} M \stackrel{\pi_{i}}{\longrightarrow} S^{p}
$$

onde $S_{2}=\{*\} \times S^{p} \times\{*\} \times \ldots \times\{*\} \subset M$.

Para $i=1,2, \ldots, n$, temos

$$
\begin{gathered}
\left(\pi_{1} \circ f_{u} \circ k_{2}\right)(*, x, *, \ldots, *)=\theta_{x}(*)=\varphi_{*}(x) \\
\left(\pi_{2} \circ f_{u} \circ k_{2}\right)(*, x, *, \ldots, *)=x
\end{gathered}
$$




$$
\begin{gathered}
\left(\pi_{3} \circ f_{u} \circ k_{2}\right)(*, x, *, \ldots, *)=* \\
\vdots \\
\left(\pi_{n} \circ f_{u} \circ k_{2}\right)(*, x, *, \ldots, *)=*
\end{gathered}
$$

Assim, $\operatorname{deg}\left(\pi_{1} \circ f_{u} \circ k_{2}\right)=\operatorname{deg}\left(\varphi_{*}\right)=2$, já que $p$ é ímpar e $a_{12}=2$, agora, $\operatorname{deg}\left(\pi_{2} \circ f_{u} \circ k_{2}\right)$ é igual ao grau da aplicação identidade, logo é 1 , e $a_{22}=1$, e os graus de $\pi_{i} \circ f_{u} \circ k_{2}$ para $i \geq 3$ são iguais a zero, $\operatorname{logo} a_{i 2}=0$ para $i \geq 3$.

Similarmente, temos quc $a_{i i}=1$ para $i \geq 30 a_{i j}=0$ paral $j \geq 3$ a $i \neq j \neq . j$ Portanuto, a matriz de representação de $\left(f_{u}\right)_{*}$ é dada por $U R$.

Usando o Lema 2.1.1 junto coin os difeomorfismos $f_{\sigma},\left(\sigma \in \mathfrak{G}_{n}\right), f_{r}, f_{l}$ e $f_{u}$, vemos que $D_{p}$ contém os subgrupos $G L(n ; \mathbb{Z}), G_{1}, G_{2}$.

Combinando os Lemas 2.1.2 e 2.1.3, vemos que $D_{p}$ está contido nos subgrupos $G L(n ; \mathbb{Z})$, $G_{1}, G_{2}$. E com isso completamos a prova do Teorema 2.2.1.

\subsection{Alguns resultados especiais}

(a) Seja $A \in G L(n ; \mathbb{Z})$ uma matriz realizada por um difeomorfismo $f: M \rightarrow M$. Entrão temos que $f$ preserva a orientação de $M$ se, e somente se,

1. $\operatorname{det} A=1$ quando $p$ é ímpar;

2. O número de entradas negativas de $A$ é par quando $p$ é par.

De fato, se $f$ preserva a orientação de $M$ sabemos então que $\operatorname{det} A=1$ para qualquer $p$, em particular para o caso em que $p$ é ímpar. Observe que no caso onde $p$ é par, pelo Lema 2.1.3, temos que $A \in G_{2}$, e como toda matriz em $G_{2}$ possui apenas um elemento não nulo (1 ou -1) em cada linha e cada coluna, concluímos que seu determinante é o produto desses elementos não nulos, e temos por hipótese que $f$ preserva a orientação de $M$, ou seja, $\operatorname{det} A=1$ então a matriz deve possuir un número par de entradas negativas.

Reciprocamente, se $\operatorname{det} A=1$ quando $p$ é ímpar, então sabemos que $f$ preserva a orientação de $M$. Agora, se quando $p$ é par o número de entradas negativas em $A$ é par, temos pelo Lema 2.1.3 que $\operatorname{det} A=1$, e logo, $f$ preserva a orientação de $M$.

(b) $D_{p}^{+}$, o grupo formado pelas matrizes que podem ser realizadas por um difcomorfismo que preserva a orientação, é um subgrupo de $D_{p}$ de índice 2 , uma véz que det é 
um epimorfismo de $D_{p}$ em $\mathbb{Z}_{2}$, onde $D_{p}^{+}$é o núcleo de det. Assim, pelo teorema do isomorfismo

$$
\frac{D_{p}}{D_{p}^{+}} \approx \mathbb{Z}_{2}
$$

e como

$$
\frac{\left|D_{p}\right|}{\left|D_{p}^{+}\right|}=\left[D_{p}: D_{p}^{+}\right] e \frac{\left|D_{p}\right|}{\left|D_{p}^{+}\right|}=\left|\mathbb{Z}_{2}\right|=2
$$

obtemos

$$
\left[D_{p}: D_{p}^{+}\right]=2
$$

Em particular, quando $p$ é ímpar e $D_{p}$ é igual a $G L(n ; \mathbb{Z})$ ou a $G_{1}$, temos que $D_{p}^{+}=$ $D_{p} \cap S L(n ; \mathbb{Z})$, onde $S L(n ; \mathbb{Z})=\{A \in M(n ; \mathbb{Z}) ; \operatorname{det} A=1\}$. Quando $p$ é par $D_{p}=G_{2}$, sabemos que $\left|G_{2}\right|=2^{n} n$ ! e $\left[G_{2}: D_{p}^{+}\right]=2$, logo $\left|D_{p}^{+}\right|=2^{n-1} n$ ! e como $G_{2}$ é isomorfo ao produto semi-direto de $\mathbb{Z}_{2}^{n}$ por $\mathfrak{G}_{n}$ temos que $D_{p}^{+}$é isomorfo a $\mathbb{Z}_{2}^{n-1}$ por $\mathfrak{G}_{n}$.

(c) Seja $\pi_{0} D i f f(M) \circ$ grupo de difeotopia de $M$, isto é, o grupo das classes de isotopia de difeomorfismos de $M$. A aplicação que associa à classe de um difeomorfismo $f$ de $M$ a matriz $I_{f}$ é o homomorfismo natural $\varphi: \pi_{0} \operatorname{Diff}(M) \rightarrow G L(n ; \mathbb{Z})$, cuja a imagem foi determinada no Teorema 2.2 .1 e o núcleo é o subgrupo $\pi_{0} S D i f f(M)$ de $\pi_{0} \operatorname{Diff}(M)$, onde $S \operatorname{Diff}(M)$ é o grupo de difeomorfismos de $M$ os quais induzem a aplicação identidade em $H_{p}(M)$, segundo Sato [18]. 


\section{Capítulo 3}

\section{Classificação dos mergulhos de esferas}

Neste capítulo, veremos uma completa classificação dos mergulhos suaves de $p$-esferas em $M$ a menos de difeomorfismos, sob certas condições sobre $n$ e $p$, veja Lucas [14].

Pelo teorema de Hurewicz, temos que $\pi_{p}(M) \cong H_{p}(M)$, logo toda classe de homologia de $H_{p}(M)$ pode ser representada por uma aplicação contínua da $p$-esfera (orientada). Então, pelo teorema de aproximação de Haefliger [9], temos

1. Se $p=1,2$ e $n \geq 3$, ou se $p \geq 3$ e $n \geq 2$, então toda classe de homologia de $H_{p}(M)$ pode ser representada por uma $p$-esfera mergulhada, suavemente.

2. Se $p=1$ e $n \geq 4$, ou $p=2,3$ e $n \geq 3$, ou $p \geq 4$ e $n \geq 2$, então duas $p$-esferas mergulhadas suavemente em $M$ representando a mesma classe de homologia são sempre suavemente isotópicas.

Observe que as condições em 2 implicam as condições em 1.

\subsection{O teorema de classificação de esferas mergulhadas no produto de esferas}

Definamos equivalência entre duas $p$-esferas mergulhadas suavemente em produto de esferas:

Definição 3.1.1. Dizemos que duas p-esferas mergulhadas suavemente (não orientadas) em $M$ são equivalentes se existe um difeomorfismo de $M$ aplicando uma na outra, onde o difeomorfismo pode não preservar a orientação.

Vejamos agora, o teorema de classificação de esferas mergulhadas: 


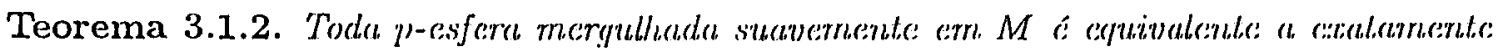
uma das seguintes p-csferas mergulhadas suavemente:

(i) Para $p=1,3,7$, uma $p$-esfera representando $(i, 0, \ldots, 0)$, com $i=0,1,2, \ldots$, $\mathrm{cm}$ $H_{p}(M) \cong \mathbb{Z}^{n}$.

(ii) Para $p$ impar com $p \neq 1,3,7$, uma $p$-esfera representando 0 ou

$$
(\underbrace{i, \ldots, i}_{j}, \underbrace{0, \ldots, 0}_{n-j}), i=1,2, \ldots ; j=1,2, \ldots, n \text {. }
$$

(iii) Para p par, uma p-esfera representando $\left(i_{1}, \ldots, i_{n}\right)$ com $0 \leq i_{1} \leq \ldots \leq i_{n}$.

Demonstração: Segundo a condição (2) de Haefliger citada acima, precisamos nos preocupar apenas com as classes de homologia dos mergulhos. Seja $S$ uma p-esfera mergulhada suavemente em $M$ representando $\alpha=\left(a_{1}, \ldots, a_{n}\right)$ em $H_{p}(M) \cong \mathbb{Z}^{n}$. Podemos assumir quo $\alpha$ é não nulo. Seja $d$ o máximo divisor comum de $a_{1}, \ldots, a_{n}$.

(i) Existe un automorfismo algébrico de $H_{p}(M)$ que leva $\alpha / d=\left(a_{1} / d, \ldots, a_{n} / d\right)$ en $(1,0, \ldots, 0)$. De fato, pensemos primeiro no caso $n=2$, onde temos $\alpha / d=\left(a_{1} / d, a_{2} / d\right)$. Como $a_{1} / d$ e $a_{2} / d$ são primos entre si, existem $r, s \in \mathbb{Z}$ tal que $r a_{1} / d+s a_{r_{2}} / d=1$. Logo, sendo

$$
A=\left(\begin{array}{cc}
a_{1} / d & -s \\
a_{2} / d & r
\end{array}\right)
$$

temos,

$$
\left(\begin{array}{cc}
a_{1} / d & -s \\
a_{2} / d & r
\end{array}\right)\left(\begin{array}{l}
1 \\
0
\end{array}\right)=\left(\begin{array}{c}
a_{1} / d \\
a_{2} / d
\end{array}\right)
$$

$\mathrm{e} \operatorname{det} A=1$. Assim

$$
A\left(\begin{array}{l}
d \\
0
\end{array}\right)=\left(\begin{array}{l}
a_{1} \\
a_{2}
\end{array}\right)
$$

Portanto

$$
A^{-1}\left(\begin{array}{l}
a_{1} \\
a_{2}
\end{array}\right)=\left(\begin{array}{l}
d \\
0
\end{array}\right)
$$

Sendo $n=3$, temos $\alpha / d=\left(a_{1} / d, a_{2} / d, a_{3} / d\right)$.

Se $a_{1} / d_{1}, a_{12} / d$ e $a_{33} / d$ não são dois a dois primos entre si, então tomemos $a_{1} / d_{1}, a_{2} / d$, tál que $m d c\left(a_{1} / d, a_{2} / d\right)=l$ e logo, $\operatorname{mdc}\left(l, a_{3} / d\right)=1$. 
Assim, existem $r_{1}, r_{2}, r_{3}, r_{4} \in \mathbb{Z}$ tais que

$$
r_{1} a_{1} / d+r_{2} a_{2} / d=l \quad \text { e } r_{3} a_{3} / d+r_{4} l=1
$$

Com isso, consideremos a seguinte matriz

$$
\left(\begin{array}{ccc}
a_{1} / d & -r_{2} & y \\
a_{2} / d & r_{1} & x \\
a_{3} / d & 1 & r_{4}
\end{array}\right)
$$

onde precisamos encontrar $x$ e $y$ de modo que $\operatorname{det} A$ seja 1 .

Temos que $\operatorname{det} A=r_{4} l-x\left(a_{1} / d+r_{2} a_{3} / d\right)+y\left(a_{2} / d-r_{1} a_{3} / d\right)$.

Considerando $x=-r_{3} a_{2} / l d$ e $y=-r_{3} a_{1} / l d$ obtemos

$$
\operatorname{det} A=r_{4} l+r_{3} a_{2} / l d\left(a_{1} / d+r_{2} a_{3} / d\right)-r_{3} a_{1} / l d\left(a_{2} / d-r_{1} a_{3} / d\right)
$$

e usando as igualdades de (1) concluímos que $\operatorname{det} A=1$.

Para verificar que $\operatorname{det} A=1$ quando consideramos $x$ e $y$ como acima podemos também olhar para a matriz $A$, que fica da forma

$$
\left(\begin{array}{ccc}
a_{1} / d & -r_{2} & -r_{3} a_{1} / l d \\
a_{2} / d & r_{1} & -r_{3} a_{2} / l d \\
a_{3} / d & 1 & r_{4}
\end{array}\right)
$$

Observe que por (1) $r_{4}=1 / l-r_{3} a_{3} / d l$ e $A$ fica da forma

$$
\left(\begin{array}{ccc}
a_{1} / d & -r_{2} & -r_{3} a_{1} / l d \\
a_{2} / d & r_{1} & -r_{3} a_{2} / l d \\
a_{3} / d & 1 & 1 / l-r_{3} a_{3} / d l
\end{array}\right)
$$

e $\operatorname{det} A$ será

$$
\operatorname{det}\left(\begin{array}{ccc}
a_{1} / d & -r_{2} & 0 \\
a_{2} / d & r_{1} & 0 \\
a_{3} / d & 1 & 1 / l
\end{array}\right)+\operatorname{det}\left(\begin{array}{ccc}
a_{1} / d & -r_{2} & -r_{3} a_{1} / l d \\
a_{2} / d & r_{1} & -r_{3} a_{2} / l d \\
a_{3} / d & 1 & -r_{3} a_{3} / d l
\end{array}\right)=1+0=1
$$

Assim

$$
A\left(\begin{array}{l}
d \\
0 \\
0
\end{array}\right)=\left(\begin{array}{l}
a_{1} \\
a_{2} \\
a_{3}
\end{array}\right)
$$


Portanto

$$
A^{-1}\left(\begin{array}{l}
a_{1} \\
a_{2} \\
a_{3}
\end{array}\right)=\left(\begin{array}{l}
d \\
0 \\
0
\end{array}\right)
$$

No caso em que $a_{1} / d, a_{2} / d$ e $a_{3} / d$ são dois a dois primos entre si, basta considerar $l=1$.

Suponhamos agora, por hipótese de indução, que existe tal automorfismo para o caso $n-1$.

Consideremos o caso onde temos $n$ elementos, ou seja, $\alpha / d=\left(a_{1} / d, \ldots, a_{n} / d\right)$. Supondo que para $n-1$ elementos o máximo divisor comum não é 1 , podemos consiclerar scın perda de generalidade que $a_{1} / d, \ldots, a_{n-1} / d$ sejam esses elementos, temos assim que $m d c\left(a_{1} / d, \ldots, a_{n-1} / d\right)=t . \quad \operatorname{Logo} m d c\left(t, a_{n} / d\right)=1$, então existem $\gamma, \beta \in \mathbb{Z}$ tál que $\gamma t+\beta a_{n h} / d l=1$.

Sendo

$$
A=\left(\begin{array}{ccccc}
a_{1} / d & b_{12} & \ldots & b_{1 n-1} & b_{1 n} \\
\vdots & \vdots & \ddots & \vdots & \vdots \\
a_{n-1} / d & b_{n-1} 2 & \ldots & b_{n-1} n-1 & b_{n-1 n} \\
a_{n} / d & 1 & \ldots & 1 & \gamma
\end{array}\right)
$$

Temos, por hipótese de indução, que conseguimos construir a matriz

$$
B=\left(\begin{array}{cccc}
a_{1} / d & b_{12} & \ldots & b_{1 n-1} \\
\vdots & \vdots & \ddots & \vdots \\
a_{n-1} / d & b_{n-1} 2 & \ldots & b_{n-1} n-1
\end{array}\right)
$$

de modo que seu determinante seja $t$.

Observe que $\gamma=\frac{1}{t}-\frac{\beta a_{n}}{d t}$.

Logo, temos que $A$ è da forma

$$
\left(\begin{array}{ccccc} 
& & & & b_{1 n} \\
& B & & & \vdots \\
& & & & U_{n-1 n} n \\
a_{n} / d & 1 & \ldots & 1 & \frac{1}{t}-\frac{\beta a_{n}}{d t}
\end{array}\right)
$$

Agora, queremos que $\operatorname{det} A$ seja 1, então considerando

$$
b_{1 n}=-\beta u_{1} / d t, b_{2 n}=-\beta a_{2} / d t, \ldots, b_{n-1}{ }_{n}=-\beta a_{n-1} / d t
$$


obtemos

$$
A=\left(\begin{array}{ccccc} 
& & & & -\beta a_{1} / d t \\
& B & & & \vdots \\
& & & & -\beta a_{n-1} / d t \\
a_{n} / d & 1 & \ldots & 1 & \frac{1}{t}-\frac{\beta a_{n}}{d t}
\end{array}\right)
$$

Logo $\operatorname{det} A$ será

$\operatorname{det}\left(\begin{array}{ccccc} & & & & 0 \\ & B & & & \vdots \\ & & & 0 \\ a_{n} / d & 1 & \ldots & 1 & 1 / t\end{array}\right)+\operatorname{det}\left(\begin{array}{ccccc} & & & & -\beta a_{1} / d t \\ & B & & \\ & & & & -\beta a_{n-1} / d t \\ a_{n} / d & 1 & \ldots & 1 & -\beta a_{n} / d t\end{array}\right)=t \cdot 1 / t+0=1$.

E assim

$$
A\left(\begin{array}{c}
d \\
0 \\
\vdots \\
0
\end{array}\right)=\left(\begin{array}{c}
a_{1} \\
a_{2} \\
\vdots \\
a_{n}
\end{array}\right)
$$

Portanto

$$
A^{-1}\left(\begin{array}{c}
a_{1} \\
a_{2} \\
\vdots \\
a_{n}
\end{array}\right)=\left(\begin{array}{c}
d \\
0 \\
\vdots \\
0
\end{array}\right)
$$

Pelo Teorema 2.2.1, temos que $D_{p}=G L(n ; \mathbb{Z})$, então o automorfismo acima é realizado por um difeomorfismo que aplica $S$ a uma $p$-esfera mergulhada suavemente em $M$ representando $(d, 0, \ldots, 0)$ em $H_{p}(M)$.

Podemos concluir disso, que $S$ é equivalente a todas as $p$-esferas mergulhadas suavemente em $M$ representado elementos de $H_{p}(M)$ cujo $m d c$ de suas coordenadas seja $d$, e como $d$ é um invariante de cada classe de equivalência, temos que as classes acima não são equivalentes umas as outras.

(ii) Lembrando que $\mathfrak{G}_{n}$ é o grupo das matrizes formadas a partir dos grupos de permutação, que $R$ é a matriz com diagonal $-1,1, \ldots, 1$ e que $G_{1}$ é o grupo das matrizes que contém exatamente um inteiro ímpar em cada uma de suas linhas e colunas, então por uma ação de $\mathfrak{G}_{n}$ e $R$ podemos transformar o elemento $\alpha / d$ em $\left(b_{1}, b_{2}, \ldots, b_{n}\right)$ de modo que $b_{1} \geq b_{2} \geq \cdots \geq b_{n} \geq 0$. 


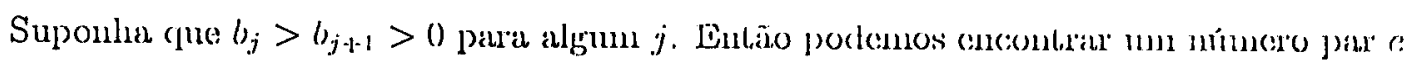
tal que

$$
\left|b_{j}-e b_{j+1}\right| \leq b_{j+1}
$$

De fato,

$$
\begin{gathered}
-b_{j+1} \leq b_{j}-e b_{j+1} \leq b_{j+1} \\
\Rightarrow-b_{j}-b_{j+1} \leq-e b_{j+1} \leq-b_{j}+b_{j+1} \\
\Rightarrow \frac{-b_{j}-b_{j+1}}{b_{j+1}} \leq-e \leq \frac{-b_{j}+b_{j+1}}{b_{j+1}} \\
\Rightarrow \frac{b_{j}+b_{j+1}}{b_{j+1}} \geq e \geq \frac{b_{j}-b_{j+1}}{b_{j+1}}
\end{gathered}
$$

e observe que $\frac{b_{j}+b_{j+1}}{b_{j+1}}$ é maior que 2 , pois se $b_{j}=n+k, b_{j+1}=n$ com $n, k \in \mathbb{Z}_{+}$, temos

$$
\frac{n+n+k}{n}=\frac{2 n+k}{n}=2+\frac{k i}{n}>2
$$

E temos também que $\frac{b_{g}-b_{j+1}}{b_{j+1}}$ é maior que 0. Logo sempre existe um par satisfazendo a desigualdade.

Agora, por uma ação de $G_{1}$ podemos transformar $\alpha / d$ em um elemento da forma $(1, \ldots, 1,0, \ldots, 0)$, pois se para algum $j$ temos $b_{j}>b_{j+1}>0$ então considerando a matriz que consiste da matriz identidade com o número par $-e$ na posição $j j+1$, onde e é o par tal que

$$
\left|b_{j}-e b_{j+1}\right| \leq b_{j+1}
$$

Chamando. essa matriz de A temos

$$
A \cdot\left(\begin{array}{c}
b_{1} \\
\vdots \\
b_{j} \\
b_{j+1} \\
\vdots \\
b_{n}
\end{array}\right)=\left(\begin{array}{c}
b_{1} \\
\vdots \\
b_{j}-e b_{j+1} \\
b_{j+1} \\
\vdots \\
b_{n}
\end{array}\right)
$$

Neste caso estamos considerando $b_{j}-e b_{j+1}=b_{j+1}$. Caso $b_{j}-e b_{j+1}<b_{j+1}$, por uma ação de $\mathfrak{G}_{n}$, e uma ação de $R$ se $b_{j}-e b_{j+1}<0$, obtemos o elemento $\left(b_{1}, b_{2}, \ldots, b_{j-1}, b_{j+1}, \ldots\right.$, $\left.b_{j}-e b_{j+1}, \ldots, b_{n}\right)$ de modo que $b_{1} \geq b_{2} \geq \ldots \geq b_{j-1} \geq b_{j+1} \geq \ldots \geq b_{j}-e b_{j+1} \geq \cdots \geq$ $b_{n} \geq 0$. 
Considerando a composição de todas as transformações em $G_{1}$ que atuam de maneira análoga a matriz $A$ sobre $\left(b_{1}, b_{2}, \ldots, b_{n}\right)$, juntamente com as ações de $\mathfrak{G}_{n}$ e $R$ se necessário, obtemos uma matriz $B$ em $G_{1}$ tal que

$$
B \cdot\left(\begin{array}{c}
b_{1} \\
b_{2} \\
b_{3} \\
\vdots \\
b_{n-1} \\
b_{n}
\end{array}\right)=\left(\begin{array}{c}
1 \\
\vdots \\
1 \\
0 \\
\vdots \\
0
\end{array}\right),
$$

onde o número de 1's é exatamente o número de $b_{i}$ 's ímpares e conseqüentemente, o número de 0 's corresponde ao número de $b_{i}$ 's pares. Podemos ver que isso ocorre quando observamos o processo de troca de $b_{j}$ por $\left|b_{j}-e b_{j+1}\right|$, este procedimento diminui os valores das coordenada, e como estamos fazendo a troca por uma combinação linear de elementos da $n$-upla, isso mantém o seu $m d c$ que é 1 , para a nova $n$-upla. Com isso, temos que a $n$-upla final não pode ser o vetor nulo pois alteraria o $m d c$. Logo, em algum momento a combinação linear dos elementos da $n$-upla será 1 , e a partir disso conseguimos trocar os ímpares por 1 e os pares por 0 .

Assim, se considerarmos o elemento $\alpha=\left(a_{1}, \ldots, a_{n}\right)$ inicial, onde $m d c\left(a_{1}, \ldots, a_{n}\right)=1$, temos pelo processo realizado acima que existe um elemento $\bar{B} \in G_{1}$ representando o difeomorfismo de $M$ que aplica $S$ em uma $p$-esfera mergulhada suavemente em $M$ representando $(1, \ldots, 1,0, \ldots, 0)$ em $H_{p}(M)$ (Lembre-se que neste caso $G_{1}=D_{p}$, que é o conjunto das matrizes de representação dos automorfismos $f_{*}: H_{p}(M) \rightarrow H_{p}(M)$ induzidos pelos difeomorfismos $f: M \rightarrow M$ ).

Observe também, que para dois elementos $\alpha=\left(a_{1}, \ldots, a_{n}\right)$ e $\beta=\left(b_{1}, \ldots, b_{n}\right)$ em $H_{p}(M)$ onde $m d c\left(a_{1}, \ldots, a_{n}\right)=m d c\left(b_{1}, \ldots, b_{n}\right)=d$ e possuem o mesmo número $j$ de coordenadas tal que $a_{i} / d$ e $b_{k} / d$ são ímpares, são levados no mesmo elemento $(\underbrace{d, \ldots, d}_{j}, \underbrace{0, \ldots, 0}_{n-j})$ por ações de $G_{1}$, ou seja $d$ e $j$ são invariantes segundo a ação de $G_{1}$. Portanto todas as classes no Teorema 3.1.2(ii) são distintas.

(iii) Quando $p$ é par temos que $D_{p}=G_{2}=<\mathfrak{G}_{n}, R>$. Logo, por uma ação de $G_{2}$, podemos transformar qualquer elemento $\alpha=\left(a_{1}, \ldots, a_{n}\right) \in H_{p}(M)$ em um elemento $\left(i_{1}, \ldots, i_{n}\right)$ com $0 \leq i_{1} \leq \ldots \leq i_{n}$, em $H_{p}(M)$.

Dado dois elementos $\alpha=\left(a_{1}, \ldots, a_{n}\right)$ e $\beta=\left(b_{1}, \ldots, b_{n}\right)$ em $H_{p}(M)$, tais que para cada 
$a_{i}$ exista um $b_{k}$ com $\left|a_{i}\right|=\left|b_{k}\right|$ e para cada $b_{k}$ exista um $a_{i}$ com $\left|a_{i}\right|=\left|b_{k}\right|$, ou seja, exista uma bijeção entre $\alpha$ e $\beta$ mantendo módulo das coordenadas, então existem matrizes $A, B \in G_{2}$ tal que $A \alpha=B \beta$, ou $B^{-1} A \alpha=\beta$. Disso podemos concluir que para um elemento $\left(a_{1}, \ldots, a_{n}\right) \in H_{p}(M)$ a $n$-upla não ordenada $\left(\left|a_{1}\right|, \ldots,\left|a_{n}\right|\right)$ é invariante segundo a ação de $G_{2}$. Portanto as classes são todas distintas. 


\section{Capítulo 4}

\section{Casos especiais}

\subsection{Classificação de esferas mergulhadas no produto de esferas, caso $n=2$}

O teorema apresentado no capítulo anterior foi provado por Goldstein [7], quando $n=2 \mathrm{e}$ $p \geq 4$, ou seja, Goldstein provou que qualquer $p$-esfera mergulhada em $S^{p} \times S^{p}$ é equivalente a um dos seguintes mergulhos suaves:

1. A esfera representando $(i, 0), i=1,2, \ldots$ em $H_{p}\left(S^{p} \times S^{p}\right)$, quando $p=3,7$,

2. Uma esfera como acima ou uma representando $(i, i), i=1,2, \ldots$, quando $p \neq 3,7$,

3. Uma esfera representando $(i, j)$ onde $0 \leq i \leq j$, quando $p$ é par e maior que 2 .

Para provar este resultado, Goldstein considera a $S^{p}$ mergulhada em $S^{p} \times S^{p}$ representando $(m, n)$ em $H_{p}\left(S^{p} \times S^{p}\right)$, excluindo os casos onde $m, n$ são nulos e considerando $d$ como o máximo divisor comum entre $m$ e $n$. Depois faz a demonstração para cada caso:

(Caso 1) Existem inteiros $a$ e $b$ tais que

$$
g=\left(\begin{array}{cc}
a & b \\
-n / d & m / d
\end{array}\right) \in G L(2 ; \mathbb{Z})
$$

então pela prop. 2.5 de Goldstein [6], existe um difeomorfismo $f$ tal que $f_{*}=g$. Com isso, $f\left(S^{p}\right)$ representa $(d, 0)$ e as classes acima são todas distintas.

(Caso 2) Se $m / d$ ou $-n / d$ é par, digamos $m / d$, então podemos encontrar inteiros $a$ e $b$, onde $a$ é par e $a m / d+b n / d=1$. Assim

$$
g=\left(\begin{array}{cc}
a & b \\
-n / d & m / d
\end{array}\right) \in G_{1}
$$


e podemos encontrar um difeomorfismo $f_{1}$ tal que $f_{1}\left(S^{p}\right)$ representa $(f l, 0)($ se $m / d$ a j $\mathrm{mr}$ então $b$ pode ser escolhido sendo par). Se $m / d$ e $n / d$ são ímpares, então podemos encontrar inteiros $a$ e $b$ tal que $a m / d+b n / d=1$. Claramente $a$ ou $b$ é par e

$$
g=\left(\begin{array}{cc}
a & b \\
a-n / d & b+m / d
\end{array}\right) \in G_{1} .
$$

Uma esfera que representa $(i, i)$ é diferente de uma que representa $(j, 0)$, já que se existisse um difeomorfismo que mandasse uma esfera sobre a outra, então em sua matriz, teríamos duas entradas $x$ e $y$ na mesma linha ou coluna, tal que $|x|=|y|$ e tal matriz não pertence a $G_{1}$.

(Caso 3) Como as entradas das matrizes em $G_{2}$ são sempre 0,1 e -1 , o único difeomorfismo que pode ocorrer, troca as posições de $m$ e $n$, ou multiplica $m$ ou $n$ por -1 . E com isso a demonstração é couscluída.

\subsection{O caso orientável}

Se considerarmos as orientações de $M$ e/ou as orientações das p-esferas mergulhadas, na Definição 3.1.1, então obteremos resultados um pouco diferentes do Teorema 3.1.2, como a seguir.

- Considerando apenas a orientação da p-esfera mergulhada e ignorando a orientação de $M$, temos pela prova do Teorema 3.1 .2 o mesmo resultado, pois se considerarmos as duas possiveis orientações, $\left(S^{p}, o r\right)$ e $\left(S^{p},-o r\right)$, existe um difeomorfismo de $M$ que leva $\left(S^{p}, o r\right)$ em $\left(S^{p},-o r\right)$. Logo elas são equivalentes, e como não estamos considerando a orientação de $M$, o resultado do teorema não se altera.

Quando $p$ é ímpar, para cada $p$-esfera na lista, existe um difeomorfismo invertendo a orientação de $M$ que preserva as classes de homologia. De fato, se $p=1,3,7$ temos pelo Teorema 3.1.2 que uma esfera $S^{p}$ mergulhada suavemente em $M$ é equivalente a $p$-esfera mergulhada representanclo $(i, 0, \ldots, 0) \operatorname{com} i=0,1,2, \ldots$, cm $H_{p}(M) \cong \mathbb{Z}^{n}$, não importando se $n$ é par ou ímpar e se a esfera $S^{p}$ está orientada ou não.

Com isso, o difeomorfismo que inverte a orientação de $M$, leva a $p$-esfera mergulhada em $M$, equivalente a $p$-esfera representando $(i, 0, \ldots, 0)$, na esfera $S^{p}$ equivalente a $p$-esfera representando $(-i, 0, \ldots, 0) \operatorname{com} i=0,1,2, \ldots$, preservando desse modo, a classe de homologia.

Agora, se $p \neq 1,3,7$, temos pelo Teorema 3.1.2 que uma esfera $S^{p}$ mergulhada suavemente 
em $M$ é equivalente a uma $p$-esfera mergulhada representando 0 ou

$$
(\underbrace{i, \ldots, i}_{j}, \underbrace{0, \ldots, 0}_{n-j}), i=0,1,2, \ldots \text { e } j=1,2, \ldots, n
$$

em $H_{p}(M)$, não importando se $n$ é par ou ímpar e se a esfera $S^{p}$ está orientada ou não.

Assim, o difeomorfismo que inverte a orientação de $M$ leva a esfera $S^{p}$ mergulhada em $M$, equivalente a $p$-esfera representando $(i, \ldots, i, 0, \ldots, 0)$, na esfera $S^{p}$ equivalente a $p$ esfera representando $(-i, \ldots,-i, 0, \ldots, 0)$ com $i=0,1,2, \ldots$, e logo, preservando a classe de homologia.

Quando $p$ é par e $n$ é ímpar, temos o mesmo resultado, se considerarmos apenas a orientação de $M$ e ignorarmos a da $S^{p}$, já que existe um difeomorfismo invertendo a orientação de $M$ que age como a multiplicação por -1 em $H_{p}(M)$, pois sendo $p$ par, temos pelo Teorema 3.1.2 que toda $p$-esfera mergulhada suavemente em $M$ é equivalente a $p$-esfera representando $\left(i_{1}, \ldots, i_{n}\right) \operatorname{com} i_{1} \leq \ldots \leq i_{n}$, não importando se $n$ é par ou ímpar e se $S^{p}$ está orientada ou não.

Sendo $n$ ímpar, temos que a matriz representando o difeomorfismo que inverte a orientação de $M$,

$$
\left(\begin{array}{cccc}
-1 & 0 & \ldots & 0 \\
0 & -1 & \ldots & 0 \\
\vdots & \vdots & \ddots & \vdots \\
0 & 0 & \ldots & -1
\end{array}\right)
$$

age multiplicando os elementos de $H_{p}(M)$ por -1 .

Seja $S$ a $p$-esfera mergulhada suavemente em $(M, o r)$ representando $\left(a_{1}, \ldots, a_{n}\right) \in H_{p}(M)$. Pela ação do difeomorfismo que inverte a orientação de $M$, temos que $S$ está mergulhada em $(M,-o r)$ representando $\left(-a_{1}, \ldots,-a_{n}\right) \in H_{p}(M)$, e pelo Teorema 3.1.2, tanto $\left(a_{1}, \ldots, a_{n}\right)$ quanto $\left(-a_{1}, \ldots,-a_{n}\right)$ são equivalentes ao elemento $\left(i_{1}, \ldots, i_{n}\right)$ com $i_{1} \leq \ldots \leq i_{n}$, em $H_{p}(M)$. Então podemos ignorar a orientação da $p$-esfera e considerar apenas a de $M$.

Falta analisarmos os casos:

Caso 1: $p$ é par, $n$ é ímpar e consideram-se as orientações de $S^{p}$ e de $M$.

Caso 2: $p$ e $n$ são pares e consideram-se as orientações de $S^{p}$ e de $M$.

Caso 3: $p$ e $n$ são pares e considera-se apenas a orientação de $M$.

No caso 1, seja $S$ uma $p$-esfera mergulhada suavemente em $M$ representando $\left(a_{1}, \ldots, a_{n}\right) \in$ $H_{p}(M)$. Ignorando, por um momento, as orientações de $S$ e $M$, teremos pelo Teorema 3.1.2 que $\left(a_{1}, \ldots, a_{n}\right)$ é equivalente ao elemento $\left(i_{1}, \ldots, i_{n}\right)$ com $i_{1} \leq \ldots \leq i_{n}$, em $H_{p}(M)$. 
Vamos considerar os casos onde $i_{1}>0$, já que no caso $i_{1}=0$ caímos no caso onde a $p$-esfera mergulhada não possui componente nesta coordenada, caindo no caso $n-1$. Agora, olhando para $\left(i_{1}, \ldots, i_{n}\right)$, considerando as orientações de $S$ e $M$, para cada coordenada negativa em $\left(a_{1}, \ldots, a_{n}\right)$, multiplicamos $i_{1}$ por -1 . Realizando este processo, estaremos corrigindo as orientações de $S$ e $M$, caso tenham mudado. Logo, se $r$ é o número de $a_{i}^{\prime} s$ negativos, então $\left((-1)^{r} i_{1}, \ldots, i_{n}\right)$, em $H_{p}(M)$ é equivalente a $\left(a_{1}, \ldots, a_{n}\right)$, quando consideramos as orientações de $S$ e $M$.

Para os casos 2 e 3 podemos usar um raciocínio análogo ao anterior. Desta maneira, torna-se necessário adicionar $p$-esferas representado $\left(i_{1}, \ldots, i_{n}\right)$ com $i_{1} \leq 0 \leq i_{2} \leq \ldots \leq i_{n} \mathrm{e}$ $\left|i_{1}\right| \leq i_{2}$. 


\section{Referências Bibliográficas}

[1] J.F. Adams, On the non-existence of elements of Hopf invariant one, Ann. of Math., (2) 72 (1960) 20-104.

[2] S.O. Ajala, Diffeomorphism groups of connected sum of a product of spheres and classification of manifolds, Internat. Math. Sci., 10 (1987) 17-33.

[3] H. Cartan, Suspension et invariant de Hopf, Exposé 5, Séminaire H. Cartan, 11e. année, 1958/59. Invariant de Hopf et opérations cohomologiques secondaires, École Normale Supérieure, Paris, 1959.

[4] P.M. Cohn, Classic Algebra, John Wiley \& Sons Ltd, 2000.

[5] G.E. Cooke, R.L. Finny, Homology of cell complexes, Princeton University Press and University of Tokio Press, Princeton, New Jersey, 1967.

[6] R.Z. Goldstein, Piecewise linear unknotting of $S^{p} \times S^{q}$ in $S^{p+q+1}$, Michigan Math. J., 14 (1967) 405-415.

[7] R.Z. Goldstein, Embedding a p-sphere in a product of p-spheres, Proc. Amer. Math. Soc. 19 (1968) 621-623.

[8] M. Greenberg, Lectures on Algebraic Topology, Northeastern University, W. A. Benjamin, Inc. New York 1967.

[9] A. Haefliger, Differentiable imbeddings, Bull. Amer. Math. Soc. 67 (1961) 109-112.

[10] P.J. Hilton, An Introduction to Homotopy Theory, Cambridge, at the University Press, 1953.

[11] M.W. Hirsch, Differential topology, Springer-Verlag, 1976. 
[12] J.P.E. Hodgson, Diffeomorphisms of some smooth metastably connected manifolds, Proc. Cambridge Philos. Soc. 71 (1972) 19-23.

[13] K. Hoffman, R: Kunze, Álgebra Linear, Editora Polígono S.A., 1971.

[14] L.A. Lucas, O. Saeki, Diffeomorphisms of a product of spheres and embedded spheres, Top. and its Aplic., 123 (2002) 471-478.

[15] L.A. Lucas, Mergulhos em codimensão um de produto cartesiano de três esferas, Tese, ICMC, Universidade de São Paulo, Junho 1998.

[16] B.R. McDonald, Linear Algebra Over Comutative Rings, Monographs Textbooks Pure Appl. Math. Vol. 87, Marcel Dekker, New York, 1984.

[17] M. Newman, Integral Matrices, Pure Appl. Math., Vol. 45, Academic Press, New York, 1972.

[18] H. Sato, Diffeomorphism groups of $S^{p} \times S^{q}$ and exotic spheres, Quart.J. Math. Oxford (2) 20 (1969) 255-276.

[19] E.H. Spanier, Algebraic Topology, McGraw-Hill Series In Higer Mathematics, 1966.

[20] N.E. Steenrod, Cohomology operations, Ann. of Math. Stud., Vol. 50, Princeton Univ. Press, Princeton, NJ, 1962, Lectures by N.E. Steenrod written and revised by D.B.A. Epstein.

[21] E.C. Turner, Diffeomorphisms homotopic to the identity, Trans. Amer. Math. Soc. 186 (1973) 489-498.

[22] C.T. Wall, Killing the middle homotopy groups of odd dimensional manifolds, Trans. Amer. Math. Soc. 103 (1962) 421-433.

[23] J.H.C. Whitehead, On adding relations to homotopy groups, Ann. of Math. (2) 42 (1941) 409-428. 